\title{
EFFICACITÉ D'UN EXUTOIRE DE DÉVALAISON ET D'UNE BARRIĖRE ACOUSTIQUE POUR LES SMOLTS DE SAUMON ATLANTIQUE (SALMO SALAR L.) À L'AMÉNAGEMENT HYDROÉLECTRIQUE DE ST. CRICQ SUR LE GAVE D'OSSAU.
}

\author{
F. TRAVADE, C. GOUYOU et N. DE FAVERI
}

EDF Division Recherche et Développement, 6 quai Watier, 78400 Chatou, France.

\section{RÉSUMÉ}

Une expérimentation a été conduite en 1996 au niveau de l'aménagement hydroélectrique de St. Cricq (gave d'Ossau) dans le but de tester deux dispositifs de dévalaison destinés à éviter le passage dans les turbines des smolts de saumon atlantique (Salmo salar L.).

Le premier, localisé au barrage, consiste en une barrière acoustique répulsive destinée à détourner les smolts vers un clapet de décharge. Le champ sonore, généré par 16 émetteurs immergés, est dans la gamme de fréquence $100-600 \mathrm{~Hz}$ avec un niveau sonore maximal de 120-130 dB.

Le deuxième dispositif, localisé à l'usine, consiste en un exutoire de surface situé en rive, à proximité immédiate de la grille de prise d'eau.

L'efficacité des deux dispositifs a été évaluée par marquage-recapture et le comportement des smolts a été suivi par radiotélémétrie.

Les passages de smolts par le clapet du barrage varient de $20 \%$ à $60 \%$; cette efficacité résulte essentiellement du débit élevé transitant par le clapet. Les tests effectués avec et sans barrière acoustique n'ont mis en évidence aucun effet significatif du champ sonore.

L'efficacité de l'exutoire du bassin de mise en charge est d'environ $80 \%$. La faible largeur de la prise d'eau $(11 \mathrm{~m})$, le faible espacement des barreaux de la grille de prise d'eau $(2,5 \mathrm{~cm})$, les conditions hydrauliques devant la grille qui guident rapidement les poissons vers l'entrée de l'exutoire, et la configuration de l'entrée de l'exutoire qui crée un écoulement à faible gradient de vitesse peuvent expliquer l'efficacité élevée de ce dispositif.

Mots-clés : dévalaison, smolt, saumon atlantique, salmo salar, exutoire de surface, barrière acoustique, usine hydroélectrique, marquage-recapture, radiotélémétrie. 


\section{EFFICIENCY OF A DOWNSTREAM BYPASS AND A BEHAVIOURAL ACOUSTIC BARRIER FOR ATLANTIC SALMON SMOLTS (SALMO SALAR L.) AT THE ST. CRICQ HYDROPOWER PLANT ON THE OSSAU RIVER (FRANCE).}

\section{ABSTRACT}

An experiment was conducted in 1996 at the St. Cricq hydropower plant on the Ossau River with a view to testing two downstream migration devices designed to prevel t Atlantic salmon smolts (Salmo salar L.) from entering the turbines.

The first, at the dam, consisted in a deterrent acoustic barrier, designed to direct smolts toward a flap gate. The sound field, generated by 16 underwater sound projectors was within the $100-600 \mathrm{~Hz}$ range with a maximum sound level of $120-130 \mathrm{~dB}$.

The second, at the powerhouse forebay was composed of a surface bypass located on the bank in the immediate vicinity of the trashrack.

The efficiency of the two devices was evaluated by means of marking-recapture, and the behaviour of the smolts was monitored by radiotelemetry.

Smolts passage over the flap gate ranged from $20 \%$ to $60 \%$; this efficiency appeared essentially due to the strong discharge through the flap gate. Tests performed with and without the acoustic barrier revealed no significant effect due to the sound field.

The efficiency of the surface bypass in the forebay was found to be about $80 \%$. This good result may be explained by the small width of the intake $(11 \mathrm{~m})$, the narrow spacing of the bars in the trashrack $(2.5 \mathrm{~cm})$, the hydraulic conditions in front of the trashrack, which rapidly guided the fish toward the entrance to the bypass, and the configuration of the bypass entrance, which created flow with a low velocity gradient.

Key-words : downstream migration, smolt, Atlantic salmon, salmo salar, surface bypass, deterrent acoustic barrier, hydropower plant, mark-recapture, radio-tracking.

\section{INTRODUCTION}

L'un des problèmes posés à la migration de dévalaison dans les cours d'eau équipés de centrales hydroélectriques est celui de la mortalité due au passage des poissons dans les turbines. Pour résoudre ce problème, divers dispositifs ont été testés à l'étranger et en France. Le principe consiste à guider les migrateurs dans un exutoire contournant les turbines à l'aide de barrières physiques (grilles de maille inférieure à la taille des poissons) ou comportementales : utilisation de stimuli répulsifs ou attractifs tels que la lumière, le son ou les champs électriques. En France, depuis quelques années, un programme d'évaluation d'exutoires de surface localisés devant les grilles de prise d'eau des centrales est en cours de réalisation pour les smolts de saumon atlantique. Le site de St. Cricq sur le gave d'Ossau offrait la possibilité de tester un tel dispositif conçu d'après les critères déterminés lors des études précédentes et s'avérait en outre favorable à la mise en œuvre 
d'une barrière comportementale destinée à empêcher le passage des smolts dans la galerie rimenée d'eau à l'usine et à les diriger vers un évacuateur de crue (clapet). Le choix s'est porté sur une barrière acoustique répulsive commercialisée par une compagnie britannique (IIish Guidance Systems Ltd). Ces deux dispositifs (exutoire de surface et barrière coustique associée à un clapet) ont été installés et testés simultanément.

\section{WATÉRIEL ET MÉTHODES}

\section{Site d'étude}

L'aménagement hydroélectrique de St. Cricq est situé dans le sud-ouest de la rance, sur le gave d'Ossau, dont le bassin versant est de $488 \mathrm{~km}^{2}$ (Figure 1). Le régime hes eaux est pluvio-nival et se caractérise par de hautes eaux en hiver et au printemps et par de basses eaux d'août à octobre. Le module interannuel est de $18 \mathrm{~m}^{3} / \mathrm{s}$. Les débits moyens des mois qui correspondent à la période de dévalaison (mars, avril et mai) sont respectivement voisins de $18 \mathrm{~m}^{3} / \mathrm{s}, 28 \mathrm{~m}^{3} / \mathrm{s}$ et $43 \mathrm{~m}^{3} / \mathrm{s}$. L'aménagement de St. Cricq (Figures 1, 2 et 4) se compose d'un barrage poids de 18 mètres de longueur et 5 mètres de hauteur, au droit duquel est située, en rive droite, une prise d'eau qui dérive dans une galerie un débit maximum de $19 \mathrm{~m}^{3} / \mathrm{s}$ vers l'usine hydroélectrique. L'évacuation des crues est assurée par un clapet de 7 mètres de large situé en rive gauche du barrage et par une vanne de fond de 7 mètres de large située en rive droite.
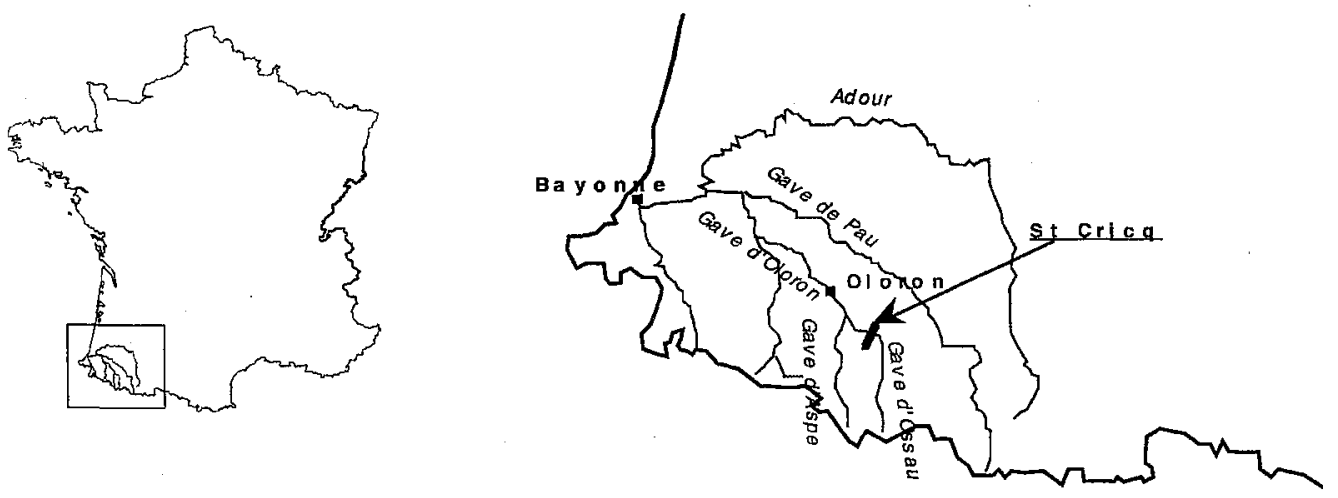

(N)

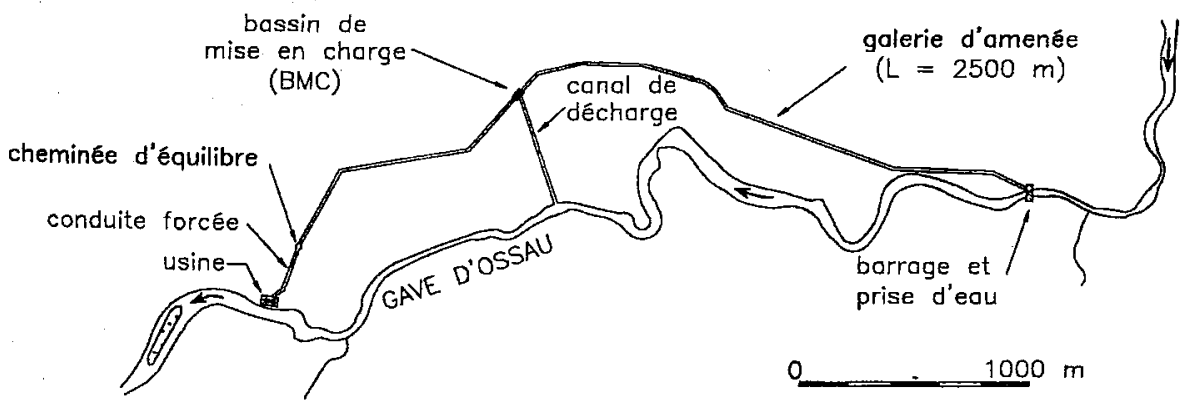

Figure 1

Situation géographique et vue générale de l'aménagement hydroélectrique de St. Cricq.

\section{Figure 1}

Geographical situation and general view of the St. Cricq hydropower plant. 
La prise d'eau se compose de trois pertuis de 3 mètres de largeur chacun et ce profondeur variant de 1,5 à 2,5 mètres suivant le niveau de la retenue (Figures 2 et 4). Dis grilles à fort écartement $(30 \mathrm{~cm})$ empêchent l'entrée des corps dérivants dans la galerie d'amenée. Une drome dirige les corps flottants vers le clapet de décharge.

La galerie d'amenée, à surface libre, de $2,5 \mathrm{~km}$ de longueur et de $12 \mathrm{~m}^{2}$ de secticn débouche dans un bassin de mise en charge (BMC) (Figures 3 et 6 ) de forme rectangulaire (e 11 mètres de large, 15,5 mètres de longueur, et de profondeur variable selon le dél it turbiné ( 3 à 4 mètres). Il est pourvu d'un déversoir de décharge et d'un plan de grilles finıs à barreaux (espacement inter-barreaux de $25 \mathrm{~mm}$ ) incliné de $35^{\circ}$ par rapport à la vertical. La surface en eau de la grille varie de $40 \mathrm{~m}^{2}$ pour le niveau bas à $59 \mathrm{~m}^{2}$ pour le nivec u haut. L'eau est entonnée dans une conduite forcée de 230 mètres de longueur cil alimente l'usine hydroélectrique sous 60 mètres de chute. L'usine, équipée de delix turbines Francis de $9,5 \mathrm{~m}^{3} / \mathrm{s}$ chacune, fonctionne au fil de l'eau.

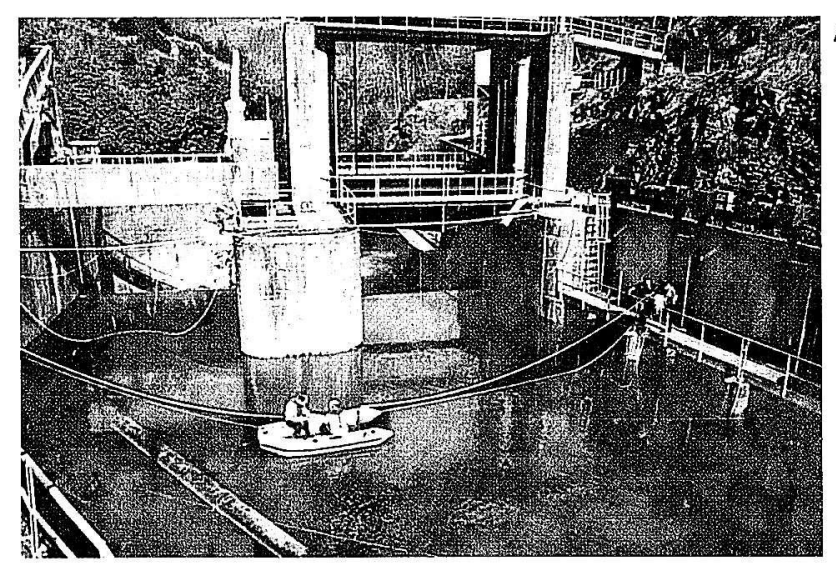

\section{A}
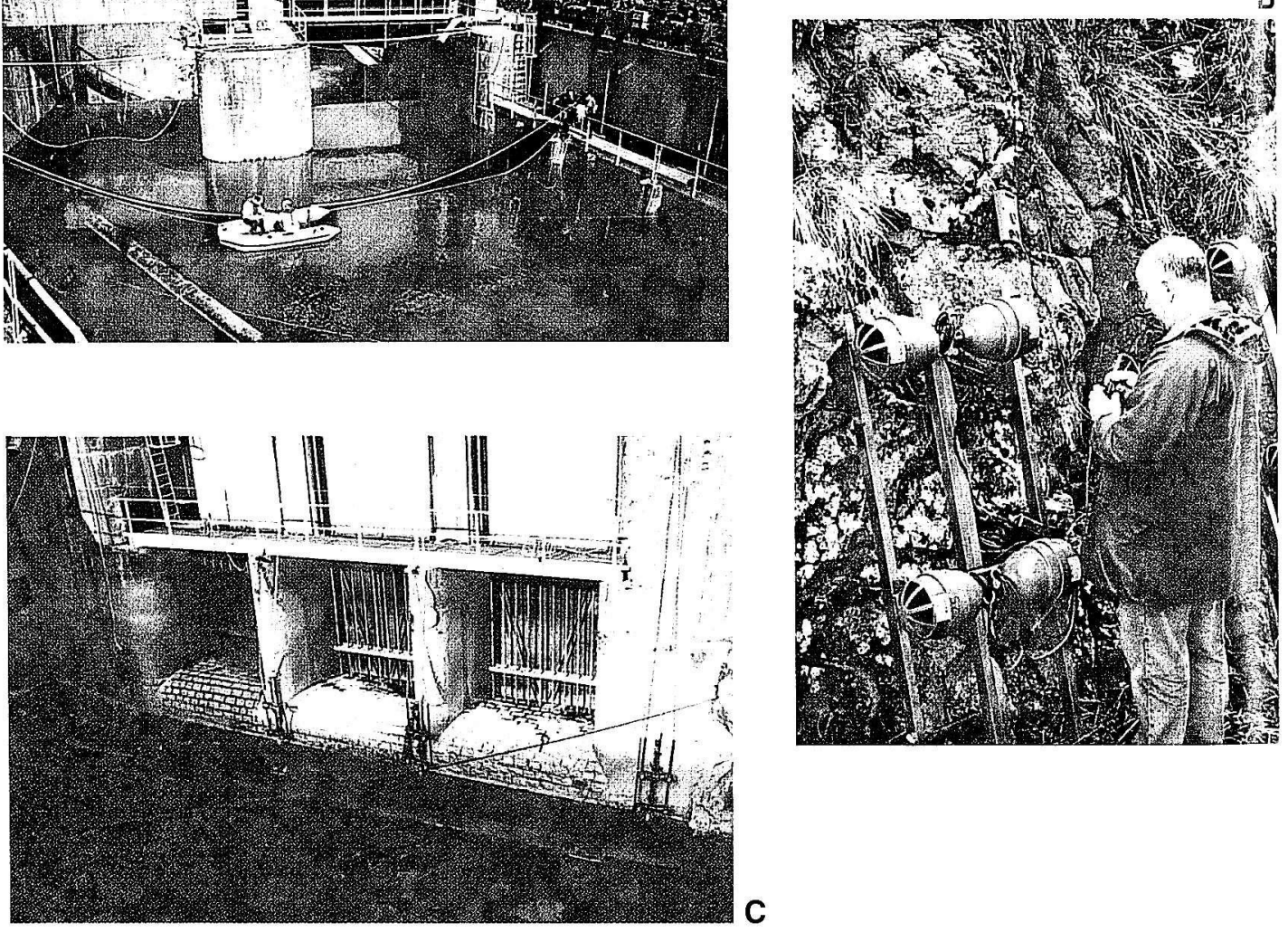

Figure 2

Barrage et retenue de St. Cricq : vue générale de la prise d'eau et du clapet de décharge (A), détail des émetteurs acoustiques subaquatiques $(B)$ et leur implantation à l'entrée de la prise d'eau (C).

Figure 2

St. Cricq dam and impoundment : general view of the intake and the flap gate $(A)$, details of the sound projectors $(B)$ and their location at the intake entrance (C). 


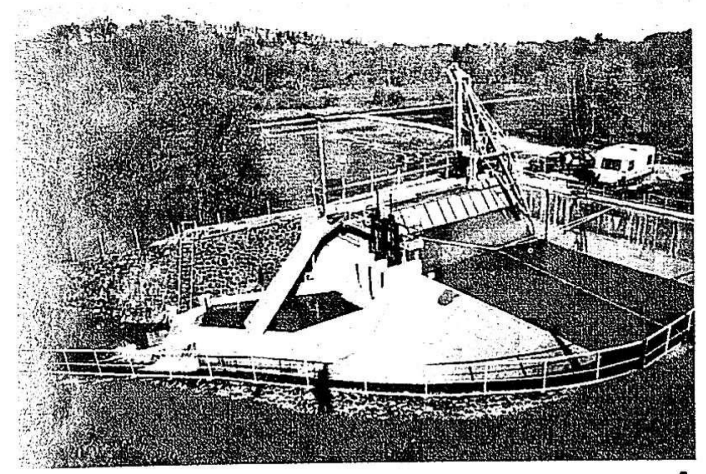

igure 3

Bassin de mise en charge (BMC) de St. Cricq : vue générale du bassin et des exutoires de dévalaison (A) et vue de l'entrée des exutoires (B).

Egure 3

St. Cricq forebay: general view of the forebay and surface bypasses (A) and detailed view of the bypass entrances $(B)$.

\section{Dispositifs de dévalaison}

Pour éviter le passage des smolts dans les turbines, deux solutions ont été testées: une barrière comportementale située au barrage et un exutoire de surface au bassin de mise en charge.

\section{Dispositif de dévalaison au barrage : clapet de décharge et barrière acoustique}

Dans son principe, le dispositif consiste à empêcher les poissons de pénétrer dans la galerie d'amenée et à les faire passer par le clapet de décharge alimenté par un débit permanent. Devant l'impossibilité d'installer une barrière physique (grille fine) à l'entrée de la galerie, il a été décidé d'utiliser une barrière comportementale répulsive destinée à éloigner les poissons de la prise d'eau (rive droite) et à les repousser en rive gauche vers le clapet.

Compte tenu de l'expérience existante sur les barrières comportementales le dispositif le plus adapté au site semblait être une barrière acoustique répulsive qui a donné des résultats intéressants lors de diverses expérimentations in situ sur des salmonidés (EPRI, 1988, 1994 ; LOEFFELMAN, VAN HASSEL et KLINECT, 1991 ; TURNPENNY, THATCHER et LOEFFELMAN, 1993 ; KNUDSEN, ENGER et SAND, 1994). La barrière est commercialisée par la firme britannique FGS (Fish Guidance Systems Ltd). Dans son principe, cette barrière est constituée d'un champ sonore (fréquences comprises entre 100 et $600 \mathrm{~Hz}$ ), diffusé dans l'eau par des émetteurs immergés, et destiné à dévier la trajectoire du poisson. FGS considère que le signal est répulsif pour les poissons si l'intensité sonore est supérieure à $20 \mathrm{~dB}$ par rapport au bruit de fond (TURNPENNY, THATCHER et LOEFFELMAN, 1993 ; TURNPENNY, 1995 ; SUBACOUSTECH, 1996a).

La localisation et la puissance des émetteurs sonores immergés ont été déterminées par FGS à l'aide d'une modélisation mathématique du champ sonore (SUBACOUSTECH, 1996a). Cette étude a conduit à l'installation de seize émetteurs sonores répartis en quatre ensembles situés à l'entrée de la prise d'eau à une profondeur de 1,7 à 3 mètres suivant la côte de la retenue (Figure 5). L'appareillage (Figure 2) se compose d'un générateur de signal (F.G.S. modèle 1-08) relié à deux amplificateurs de puissance (F.G.S. modèle 400, puissance 455 W RMS à 4 ohms) qui alimentent chacun 8 émetteurs subaquatiques (model 15, puissance $100 \mathrm{~W}$ RMS, niveau sonore à la source $164 \mathrm{~dB}$ re $1 \mu \mathrm{Pa} @ 1 \mathrm{~m})$. 
Deux séries d'amplificateurs de puissances différentes ont été testées (différence ce potentiel en sortie respectivement de 20 volts, et de 40 volts). La différence de nivéiu sonore entre les deux amplificateurs est de $10 \mathrm{~dB}$. Des mesures de répulsivité de la barrière sonore ont été effectuées avec les deux amplificateurs, dénommés par la suite " barrière 20 volts " (faible puissance) et « barrière 40 volts " (forte puissance).

Des mesures du champ sonore ont été réalisées par FGS (SUBACOUSTEC 1 , $1996 b$ ) après mise en service de l'installation (Figure 5). Ces mesures ont mis en évidence que le champ sonore généré était notablement inférieur à celui calculé par le modèle ave un maximum (respectivement pour la barrière 20 volts et 40 volts) de 20 à $30 \mathrm{oB}$ au-dessus du bruit de fond (100 dB) à̀ l'entrée de la prise d'eau (au lieu de 60 à $70 \mathrm{cB}$ escomptés) et de l'ordre de 10 à $25 \mathrm{~dB}$ au niveau de la drome.
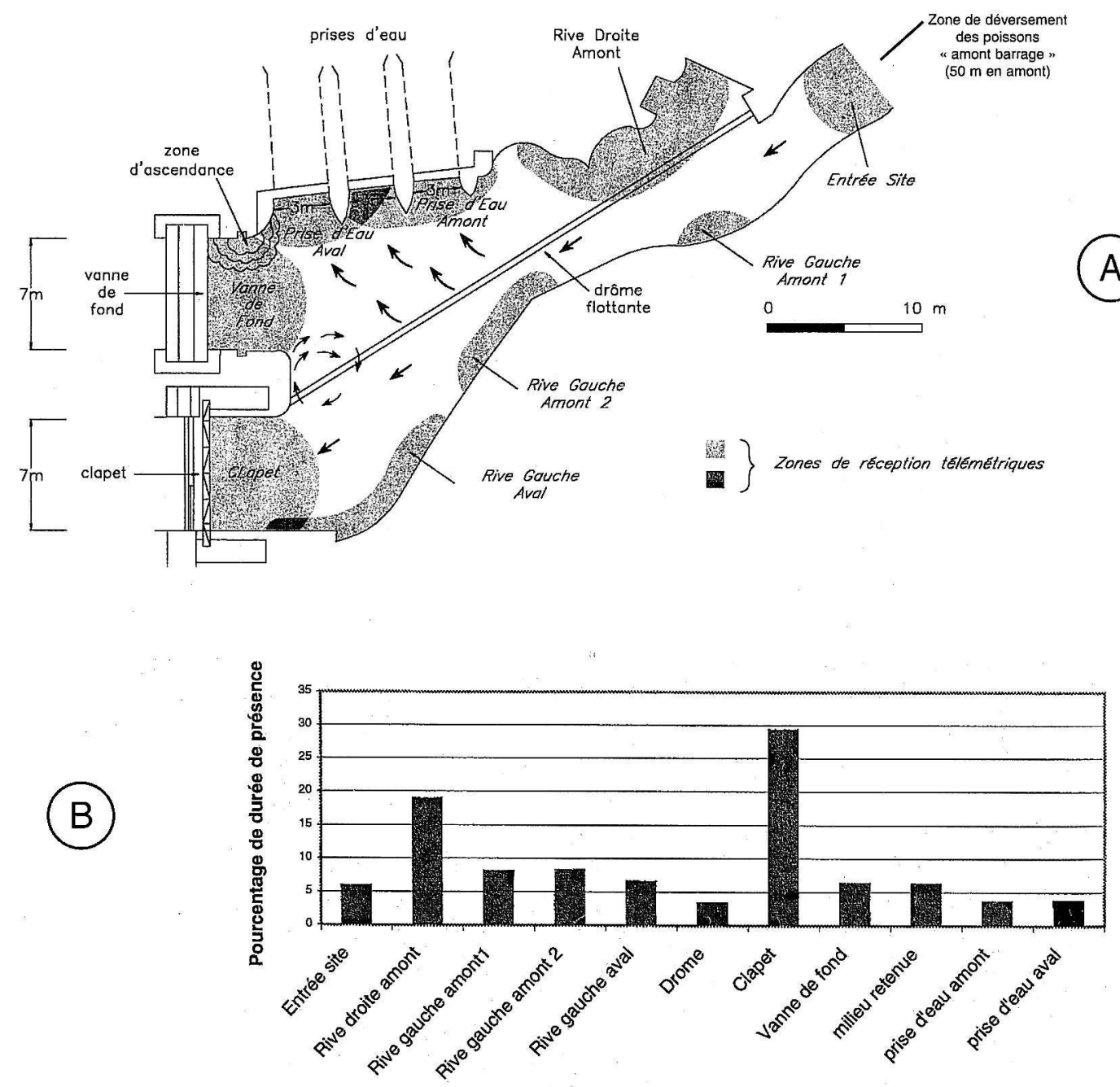

Zones

Figure 4

Vue générale, hydrodynamique, zones de réception des radioémetteurs $(A)$ et répartition du temps de présence des smolts dans les diverses zones de la retenue de St. Cricq $(B)$.

\section{Figure 4}

General view, hydrodynamics, radiotelemetry listening areas (A) and distribution of residence time for radiotagged smolts in the different zones of St. Cricq reservoir (B). 


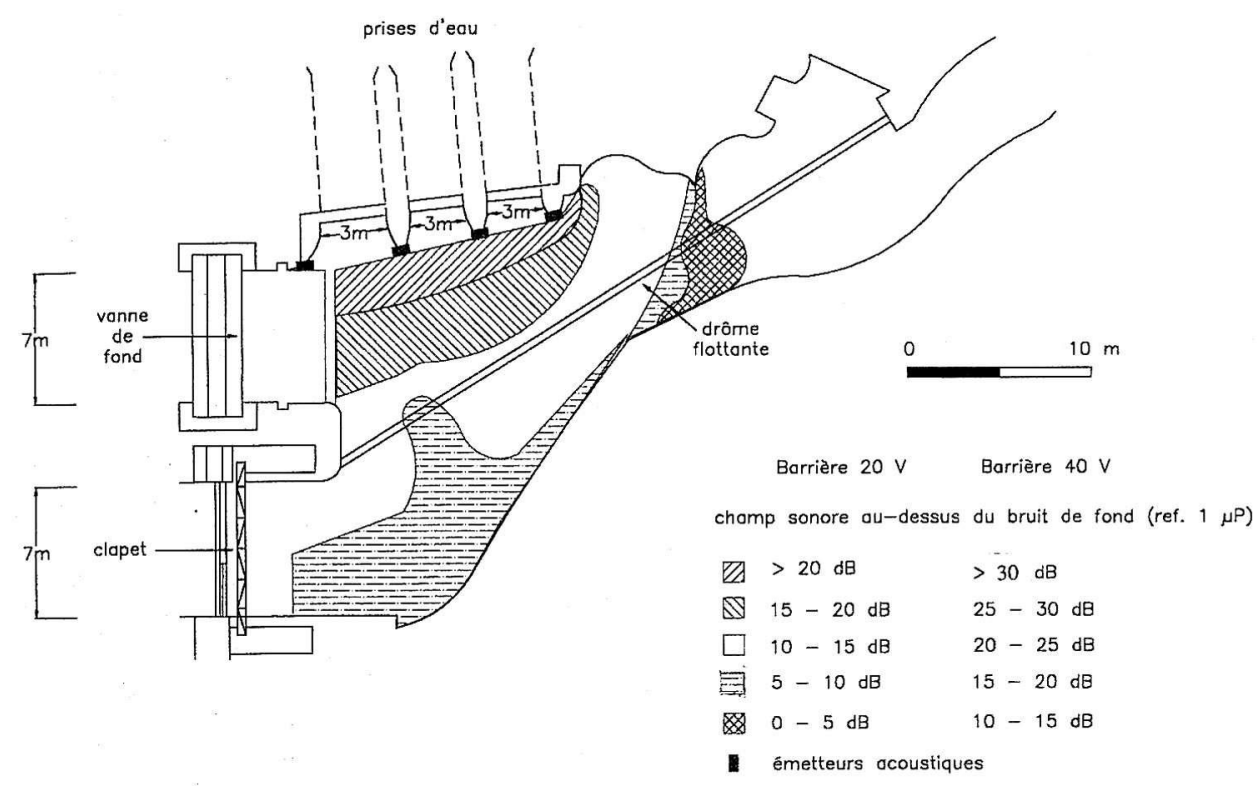

Figure 5

Implantation de la barrière acoustique et mesures du champ sonore dans la retenue de St. Cricq.

\section{Figure 5}

\section{Location of the acoustic barrier and measurements of the acoustic field in St. Cricq reservoir.}

Le clapet devant servir de by-pass pour les poissons est automatique (asservissement au niveau de la retenue par flotteurs) et déverse lorsque le débit du gave est supérieur au débit turbiné. Une manoeuvre manuelle avait donc été prévue pour délivrer un débit permanent de 1 à $3 \mathrm{~m}^{3} / \mathrm{s}$ en dehors des périodes de déversement, mais. en pratique, du fait de la très grande variabilité du débit déversé, cette manœuvre manuelle n'a pas pu être effectuée et il n'y a pas eu de déversement au clapet pendant une partie de l'expérimentation.

\section{Dispositif de dévalaison au BMC : exutoire associé à la grille de prise d'eau}

Le dispositif de dévalaison situé au BMC est constitué d'un exutoire de surface associé à la grille de prise d'eau voisin de ceux testés sur d'autres sites en France (LARINIER et BOYER-BERNARD, 1991b ; LARINIER et TRAVADE, 1996, 1997 ; GOSSET et al., 1998 ; CHANSEAU, LARINIER et TRAVADE, 1999 ; CROZE, CHANSEAU et LARINIER, 1999).

Du fait de la régulation du niveau du plan d'eau du BMC à deux cotes différentes suivant le mode de fonctionnement des turbines (un ou deux groupes en service) il a été réalisé deux exutoires juxtaposés de 0,8 mètre de large avec des seuils déversants calés en altitude pour délivrer environ $2 \%$ à $5 \%$ du débit turbiné à chacune des cotes (Figures 3 et 6). Une vanne automatique ferme l'exutoire « bas » lorsque le niveau dans le BMC est à la cote supérieure. Ces deux exutoires, situés à proximité immédiate de la grille sur le bajoyer rive gauche du BMC débouchent, par l'intermédiaire d'un seuil épais (longueur de 1 mètre), dans un bassin de réception ( $6 \mathrm{~m} \times 4,5 \mathrm{~m}$ ) comportant à son extrémité aval un déversoir de régulation du débit de 2,6 mètres de large et un dispositif de piégeage des poissons dévalants. L'entonnement a été profilé (bords arrondis) de façon à améliorer l'écoulement à l'entrée de l'exutoire. 

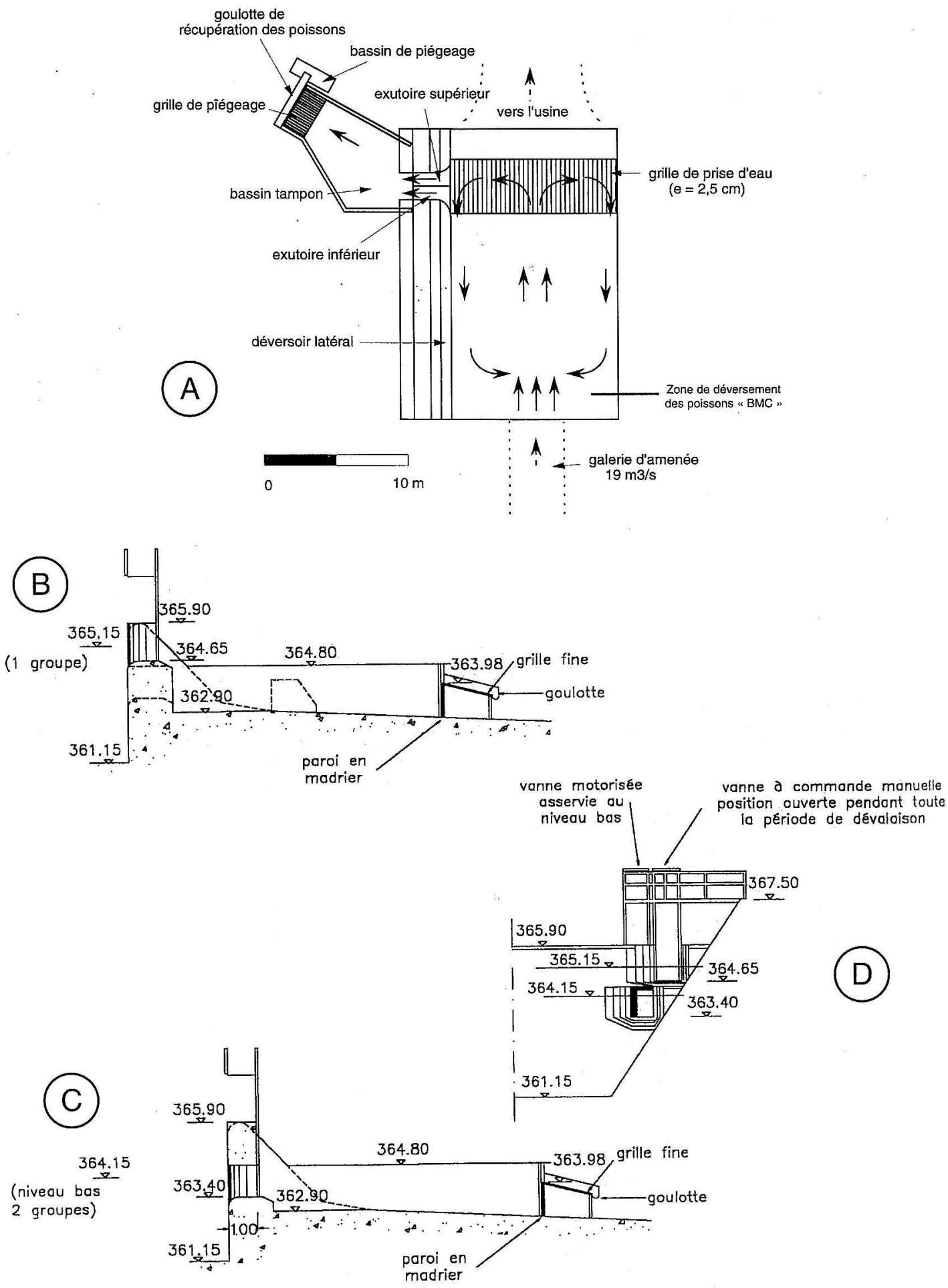

Figure 6

Vue générale et hydrodynamique du bassin de mise en charge (BMC) de St. Cricq. Vue en plan (A), coupes longitudinales $(B, C)$ et transversales (D) des exutoires de dévalaison et du dispositif de piégeage.

\section{Figure 6}

General view and hydrodynamics of St. Cricq forebay. Plane view (A) and section views $(B, C, D)$ of the downstream migration bypass and trapping facilities. 


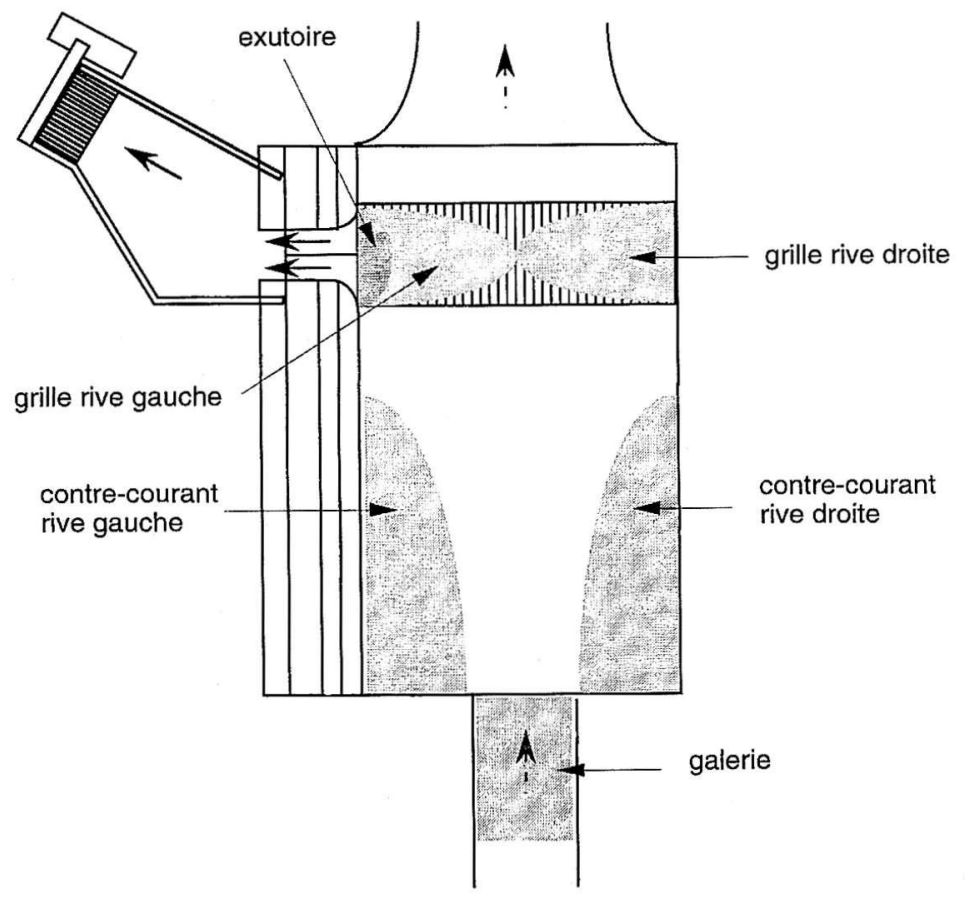

\section{Figure 7}

Zones de réception des radioémetteurs au BMC.

\section{Figure 7}

Radiotelemetry listening areas in the forebay.

L'effet attractif de la lumière (lampes à vapeur de mercure de faible puissance) sur les smolts ayant été mis en évidence lors de nombreuses études (TAFT, 1988 ; LARINIER et BOYER-BERNARD, 1991a ; EPRI, 1988, 1994 ; GOSSET et TRAVADE, 1999), l'entrée de l'exutoire a été systématiquement éclairée par une lampe à vapeur de mercure de 50 watts disposée à environ trois mètres au-dessus du niveau de l'eau. II s'agit d'un éclairage discontinu composé de phases éclairées de dix minutes et de phases éteintes de cinq minutes.

\section{Piège de contrôle des poissons dévalants}

Le piège de dévalaison est situé à l'aval du bassin de réception de l'exutoire du BMC (Figures 3 et 6 ). Il est constitué d'une grille fine (espacement des barreaux de $1 \mathrm{~cm}$ ) inclinée $(15 \%)$, de 1,45 mètre de long sur 2, 60 mètres de large, fixée sur le déversoir du bassin et prolongée d'une goulotte transversale en tôle qui guide les poissons vers une cuve.

\section{Période d'étude}

L'étude s'est déroulée du 18 mars au 25 mai 1996 (68 jours), période habituelle de migration des smolts sur ce bassin (BOUSQUET, 1979).

\section{Hydrodynamique du site}

L'hydrodynamique au voisinage des dispositifs de dévalaison a été appréciée par des mesures de vitesse d'écoulement (courantomètre Flow Mate 2000), des lâchers de flotteurs et des injections de colorant (fluorescéine). 


\section{Paramètres du milieu}

Cinq paramètres du milieu susceptibles d'influer sur la migration des smolts ou sur : fonctionnement des dispositifs de dévalaison ont fait l'objet d'enregistrements continus : a température de l'eau au barrage et au BMC, le niveau dans la retenue et la position ciu clapet de décharge au barrage destinée à calculer le débit déversé et le niveau dans BMC pour l'évaluation du débit dans l'exutoire. Ces paramètres ont été enregistrés au pes de temps de 5 minutes sur deux centrales d'acquisition portables de type Logicap (centrales CR2M SAB 600 LUS-IP). L'enregistrement graphique des débits turbinés a éá obtenu auprès de l'usine de St. Cricq. Parallèlement, ont été contrôlées à heures fixes au BMC, la conductivité de l'eau (conductimètre portable WTW) et la turbidité au disque de Secchi.

\section{Mesure d'efficacité des dispositifs}

L'efficacité des deux dispositifs a été évaluée par marquage-recapture avec une méthodologie semblable à celle utilisée sur les autres études françaises précédemment citées (LARINIER et BOYER-BERNARD, 1991b ; LARINIER et TRAVADE, 1996, 1997 ; GOSSET et al., 1998 ; CHANSEAU, LARINIER et TRAVADE, 1999 ; CROZE, CHANSEAU et LARINIER, 1999). Nous avons utilisé des smolts de pisciculture dans la mesure où les effectifs de smolts sauvages étaient très faibles en 1996 sur le gave d'Ossau.

Les poissons ont été lâchés par lots d'une centaine d'individus mesurés et marqués au préalable par ablation partielle de nageoires et/ou injection de colorant (bleu alcyan) dans une nageoire. Les combinaisons de marquage ont permis d'individualiser chacun des lots déversés.

Les déversements ont été effectués simultanément en deux points : zone "amont barrage » située une centaine de mètres en amont du barrage (Figure 4) et zone «BMC " (Figure 6) au débouché de la galerie d'amenée dans le BMC. Ils ont été réalisés en fin d'après-midi ou en début de nuit, période d'activité prépondérante des smolts dans le milieu naturel (BAGLINIERE, 1976 ; THORPE, 1981 ; LARINIER et BOYER-BERNARD, 1991a ; MCCORMICK et al., 1998), après acclimatation de plusieurs heures des poissons en bassin (zone " amont barrage ") ou en cage flottante (zone « BMC »).

2911 smolts répartis en 30 lots (16 au BMC et 14 au barrage) ont été déversés à raison de 2 lâchers par semaine.

Les poissons ont été recapturés dans le piège situé à l'aval de l'exutoire du BMC qui a été relevé trois fois par jour durant toute la campagne ( $9 \mathrm{~h}, 14 \mathrm{~h}$ et $20 \mathrm{~h}$ ).

L'efficacité de l'exutoire du BMC a été déterminée, pour chaque lot lâché dans le $B M C$, par le pourcentage de poissons recapturés dans le piège, en faisant l'hypothèse que les smolts ne remontent pas dans la retenue d'où ils pourraient passer par le clapet du barrage. Cette hypothèse semble justifiée au vu de la longueur importante de la galerie $(2,5 \mathrm{~km})$ et de la vitesse d'écoulement élevée $(1,6 \mathrm{~m} / \mathrm{s})$.

L'efficacité de la barrière acoustique associée au clapet du barrage a été évaluée à partir des captures dans le piège du BMC, car, en raison des débits déversés élevés, il était impossible de capturer les poissons transitant par le barrage. Cette évaluation se fait à partir du nombre de poissons recapturés provenant du lot " amont barrage » et de l'efficacité de l'exutoire du BMC pour le lot correspondant lâché au BMC. 
L'expression donnant l'efficacité de la barrière acoustique pour le lot « $\mathrm{i}$ » est la Survante :

$$
\mathrm{E}_{\mathrm{BA}}(\mathrm{i})=100 *\left[1-\left(100 * \frac{\mathrm{P}(\mathrm{i})}{\mathrm{L}(\mathrm{i}) * \mathrm{E}_{\text {exu }}(\mathrm{i})}\right)\right] \text {; }
$$

avec :

$E_{\mathrm{BA}}$ efficacité du clapet et de la barrière acoustique associée (\%);

$E_{\text {exu: }}$ efficacité de l'exutoire du BMC pour le lot $i$ «BMC» $(\%)$; ENC ;

$P(i)$ : nombre de poissons du lot $i$ « amont barrage " recapturés dans le piège du

L (i) : nombre de poissons composant le lot $\mathrm{i}$ « amont barrage ».

\section{Suivi du comportement des smolts}

En complément du marquage-recapture, le comportement des smolts au voisinage des dispositifs de dévalaison a été suivi par radiotélémétrie.

La technique est similaire à celle utilisée sur les autres sites précédemment cités pour les smolts de saumon atlantique (Salmo salar) et de truite de mer (Salmo trutta) (INGENDAHL et al., 1996). Elle a consisté à implanter des émetteurs radio sur un certain nombre de smolts et à suivre leurs déplacements par détection de présence dans des zones calibrées.

Le matériel utilisé, d'origine américaine, est commercialisé par Advanced Telemetry Systems (ATS). Les émetteurs d'un poids de 1,3 g et d'une durée de vie d'une douzaine de jours ont une fréquence radio individualisée dans la gamme $48-49 \mathrm{MHz}$. Ils ont été implantés dans l'estomac par voie buccale. La détection de présence dans les zones délimitées par des antennes réceptrices immergées a été réalisée automatiquement par des récepteurs enregistreurs (ATS 2100 et Data Collection D5040). Neuf zones de réception ont été délimitées en amont du barrage (Figure 4), 5 au BMC (Figure 7) et 2 à l'aval du barrage et de l'usine pour confirmer les zones de passage des poissons. Des repérages manuels à l'aide de récepteurs portables ont complété ces enregistrements automatiques.

\section{RÉSULTATS}

\section{Débits et autres paramètres de milieu}

Le débit du gave a varié, au cours de l'expérimentation, de $11 \mathrm{~m}^{3} / \mathrm{s}$ à $60 \mathrm{~m}^{3} / \mathrm{s}$ avec une moyenne de $27 \mathrm{~m}^{3} / \mathrm{s}$. Les variations journalières, résultant du fonctionnement de centrales hydroélectriques situées en amont, peuvent être très élevées (passage de $10 \mathrm{~m}^{3} / \mathrm{s}$ à plus de $30 \mathrm{~m}^{3} / \mathrm{s}$ au cours d'une même journée), entraînant de ce fait des variations importantes du débit déversé au clapet du barrage. 
Le débit déversé au clapet a varié de 0 à $40 \mathrm{~m} / \mathrm{s}$. La distribution en fréquence montre qu'il y a absence de déversement durant $18 \%$ du temps et que l'occurrence moyenne (50\% du temps) est proche de $8 \mathrm{~m}^{3} / \mathrm{s}$, correspondant à $45 \%$ du débit turbiné.

Le débit turbiné a varié de $10 \mathrm{~m} 3 / \mathrm{s}$ à $19 \mathrm{~m}^{3} / \mathrm{s}$, débit maximal de l'installation, qui a été atteint pendant $80 \%$ du temps.

Le débit dans l'exutoire de dévalaison du BMC a varié de $0,3 \mathrm{~m}^{3} / \mathrm{s}$ à $0,8 \mathrm{~m}^{3} / \mathrm{s}(1,5 \%$ à $4,2 \%$ du débit turbiné) sous l'effet des variations de niveau dans le BMC. Le débit moyen est de $0,65 \mathrm{~m}^{3} / \mathrm{s}$ correspondant à $3,4 \%$ du débit turbiné.

La température de l'eau a varié de $5,9^{\circ} \mathrm{C}$ à $10,6^{\circ} \mathrm{C}$, (moyenne de $7,5^{\circ} \mathrm{C}$ ) avec des variations nycthémérales pouvant atteindre $3^{\circ} \mathrm{C}$. La conductivité de l'eau (valeur à $20^{\circ} \mathrm{C}$ ) a varié de 155 à $234 \mu \mathrm{S} / \mathrm{cm}$ et la transparence au disque de Secchi de 0,6 mètre à 3 mètres.

\section{Hydrodynamique}

Au barrage (Figure 4), la courantologie en surface est très variable selon le débit déversé au clapet et la cote de la retenue. Lorsque le clapet déverse avec un débit supérieur à $10 \mathrm{~m}^{3} / \mathrm{s}$ environ, le courant longeant la rive gauche devient prépondérant par rapport à celui dirigé vers la prise d'eau en rive droite. On observe des courants de recirculation plus ou moins importants à l'extrémité aval de la drome et des ascendances en rive droite du barrage. Les vitesses d'écoulement mesurées en sub-surface dans les pertuis de prise d'eau sont hétérogénes et varient de 0,3 à $0,8 \mathrm{~m} / \mathrm{s}$.

Au BMC (Figure 6), la courantologie est caractérisée par un écoulement central à forte vitesse $(1,6$ à $1,8 \mathrm{~m} / \mathrm{s})$ au débouché de la galerie qui génère deux courants de recirculation symétriques très marqués. L'écoulement devant la grille est caractérisé, en surface, par des courants tangentiels orientés du centre de la grille vers les berges avec des vitesses élevées $(0,65$ à $0,8 \mathrm{~m} / \mathrm{s})$.

Les vitesses moyennes en sub-surface à l'entrée de l'exutoire pour le niveau d'eau et le débit moyens représentatifs de la gamme de variation au cours de l'expérimentation sont hétérogènes et varient de $1,1 \mathrm{~m} / \mathrm{s}$ en rive droite à $0,48 \mathrm{~m} / \mathrm{s}$ en rive gauche. Ces vitesses sont du même ordre de grandeur que celles de l'écoulement transversal devant les grilles de prises d'eau. La chute entre le niveau du BMC et le niveau du bassin tampon de l'exutoire est faible : $3 \mathrm{~cm}$ à $10 \mathrm{~cm}$ suivant le niveau du BMC.

L'exutoire apparaît hydrauliquement très attractif : lors de lâchers de flotteurs dans le BMC, $50 \%$ des flotteurs rentrent dans l'exutoire dans les 10 minutes suivant le lâcher après un ou deux passages le long de la grille de prise d'eau et la totalité y passe en 2 à 3 heures après un nombre plus ou moins important de rotations dans les contre-courants de la rive droite et de la rive gauche.

\section{Efficacité de l'exutoire de dévalaison et comportement des smolts au BMC}

\section{Efficacité de l'exutoire}

1539 smolts répartis en 16 lots d'une centaine de poissons ont été déversés dans le BMC. 1216 ont été repris dans le piège de l'exutoire, ce qui correspond à une efficacité globale de l'exutoire de $79 \%$. Cette efficacité varie selon les lots de $63 \%$ à $100 \%$ (Tableau I). La durée nécessaire au passage des poissons dans l'exutoire est également variable selon les lots : de $2 \mathrm{~h}$ à $14 \mathrm{~h} 30$ pour assurer $50 \%$ du passage et de $2 \mathrm{~h} 15$ à 14 jours pour $90 \%$ du passage. 


\section{Tableau I}

Récapitulatif des opérations de marquage-recapture (« lots BMC ") et résultats d'efficacité de l'exutoire du BMC.

\section{oble I}

Summary of mark-recapture operations ("BMC batches ") and results of forebay bypass efficiency.

\begin{tabular}{|c|c|c|c|c|c|c|c|c|}
\hline Lot & $\begin{array}{l}\text { Date } \\
\text { de } \\
\text { Lâcher }\end{array}$ & $\begin{array}{l}\text { Lâcher } \\
\text { Nombre }\end{array}$ & $\begin{array}{l}\text { Recapture } \\
\text { Nombre }\end{array}$ & $\begin{array}{c}\text { Efficacité } \\
\text { Exutoire } \\
\%\end{array}$ & $\begin{array}{l}\text { Longueur } \\
\text { moyenne } \\
\mathrm{cm}\end{array}$ & $\begin{array}{c}\text { Facteur de conditon } \\
\text { moyen }\end{array}$ & $\begin{array}{c}\text { Durée } \\
\text { recapture } \\
50 \%\end{array}$ & $\begin{array}{c}\text { Durée } \\
\text { recapture } \\
90 \%\end{array}$ \\
\hline $1 \mathrm{~A}$ & 28/3/96 & 100 & 70 & 70,0 & 17,52 & 0,87 & $12 \mathrm{~h}$ & $14 \mathrm{j} 21 \mathrm{~h}$ \\
\hline $2 \mathrm{~A}$ & $2 / 04 / 96$ & 100 & 78 & 78,0 & 17,86 & 0,83 & $12 \mathrm{~h} 30$ & $8 \mathrm{j} 21 \mathrm{~h} 30$ \\
\hline $3 A$ & $4 / 04 / 96$ & 99 & 71 & 71,7 & 17,85 & 0,85 & $12 h$ & $6 j 15 h$ \\
\hline $4 \mathrm{~A}$ & $9 / 04 / 96$ & 99 & 62 & 62,6 & 17,97 & 0,85 & 2j $3 \mathrm{~h}$ & 3j $18 \mathrm{~h}$ \\
\hline $5 \mathrm{~A}$ & $11 / 04 / 96$ & 99 & 79 & 79,8 & 19,9 & 0,81 & $2 h 30$ & $1 \mathrm{j} 22 \mathrm{~h}$ \\
\hline $6 \mathrm{~A}$ & $16 / 04 / 96$ & 100 & 83 & 83,0 & 18,1 & 0,81 & $14 h 30$ & $14 h 30$ \\
\hline $7 \mathrm{~A}$ & $18 / 04 / 96$ & 100 & 96 & 96,0 & 18,24 & 0,81 & $3 h 30$ & $17 h 30$ \\
\hline $8 \mathrm{~A}$ & $23 / 04 / 96$ & 100 & 74 & 74,0 & 17,74 & 0,88 & $14 \mathrm{~h}$ & 2j $14 \mathrm{~h}$ \\
\hline $9 \mathrm{~A}$ & $25 / 04 / 96$ & 100 & 96 & 96,0 & 18,30 & - & $2 h$ & $17 \mathrm{~h}$ \\
\hline $10 \mathrm{~A}$ & $29 / 04 / 96$ & 103 & 85 & 82,5 & 18,36 & 0,77 & 3 h30 & $17 \mathrm{~h}$ \\
\hline $11 \mathrm{~A}$ & $30 / 04 / 96$ & 144 & 129 & 89,6 & 18,35 & 0,78 & $14 \mathrm{~h}$ & $14 h$ \\
\hline $12 \mathrm{~A}$ & $2 / 05 / 96$ & 100 & 72 & 72,0 & 18,24 & 0,89 & $14 h 30$ & $6 \mathrm{j} 14 \mathrm{~h} 30$ \\
\hline $13 \mathrm{~A}$ & $8 / 05 / 96$ & 95 & 60 & 63,2 & 18,24 & 0,83 & $15 h 15$ & $15 \mathrm{~h} 15$ \\
\hline $14 \mathrm{~A}$ & $9 / 05 / 96$ & 100 & 75 & 75,0 & 18,39 & 0,85 & $14 \mathrm{~h} 30$ & $14 \mathrm{~h} 30$ \\
\hline $15 A$ & $15 / 05 / 96$ & 50 & 50 & 100,0 & 18,62 & - & 2h15 & 2h15 \\
\hline $16 \mathrm{~A}$ & $16 / 05 / 96$ & 50 & 36 & 72,0 & 18,08 & 0,85 & $15 \mathrm{~h}$ & $15 \mathrm{~h}$ \\
\hline
\end{tabular}

\section{Tableau II}

Récapítulatif des opérations de marquage-recapture (" lots Barrage ") et résultats d'efficacité du clapet associé à la barrière acoustique.

\section{Table II}

Summary of mark-recapture operations (« Dam batches ») and efficiency of flap gate combined with acoustic barrier.

\begin{tabular}{|c|c|c|c|c|c|c|c|c|c|c|}
\hline Lot & $\begin{array}{l}\text { Date } \\
\text { de } \\
\text { Lâcher }\end{array}$ & $\begin{array}{c}\text { Barrière } \\
\text { acoustique }\end{array}$ & $\begin{array}{l}\text { Lâcher } \\
\text { Nombre }\end{array}$ & $\begin{array}{c}\text { Recapture } \\
\text { Nombre }\end{array}$ & $\begin{array}{c}\text { Efficacit } \\
\text { Clapet } \\
\%\end{array}$ & $\begin{array}{c}\text { Longueur } \\
\text { moyenne } \\
\mathrm{cm}\end{array}$ & $\begin{array}{c}\text { Facteur de } \\
\text { condition } \\
\text { moyen }\end{array}$ & $\begin{array}{c}\text { Débit au clapet } \\
\text { occurrence } 50 \% \\
\mathrm{~m}^{3} / \mathrm{s}\end{array}$ & $\begin{array}{c}\text { Durée } \\
\text { recapture } \\
50 \% \\
\end{array}$ & $\begin{array}{c}\text { Durée } \\
\text { recapture } \\
90 \% \\
\end{array}$ \\
\hline $2 \mathrm{~B}$ & $2 / 04 / 96$ & $20 \mathrm{~V}$ & 100 & 30 & 61,5 & 17,84 & 0,86 & 6,3 & 13h30 & $8 \mathrm{j} 22 \mathrm{~h} 30$ \\
\hline 3B & $4 / 04 / 96$ & $20 \mathrm{~V}$ & 99 & 55 & 22,5 & 17,82 & 0,86 & 1,3 & $4 \mathrm{j} 18 \mathrm{~h} 30$ & $8 \mathrm{j} 13 \mathrm{~h} 30$ \\
\hline $4 \mathrm{~B}$ & $9 / 04 / 96$ & $E$ & 98 & 52 & 15,3 & 17,95 & 0,81 & 0,7 & $14 \mathrm{~h}$ & $2 \mathrm{j} 15 \mathrm{~h} 30$ \\
\hline $5 \mathrm{~B}$ & $11 / 04 / 96$ & E & 100 & 62 & 22,3 & 17,97 & 0,8 & 2,2 & $17 \mathrm{~h}$ & $1 \mathrm{j} 22 \mathrm{~h}$ \\
\hline $6 B$ & $16 / 04 / 96$ & $20 \mathrm{~V}$ & 102 & 36 & 57,5 & 18,07 & 0,83 & 14,9 & $14 h 30$ & $14 h 30$ \\
\hline $7 \mathrm{~B}$ & $18 / 04 / 96$ & $20 \mathrm{~V}$ & 100 & 31 & 67,7 & 18,27 & 0,81 & 11,8 & $3 h 15$ & $17 \mathrm{~h} 30$ \\
\hline $8 \mathrm{~B}$ & $23 / 04 / 96$ & $40 \mathrm{~V}$ & 100 & 35 & 52,7 & 17,98 & 0,84 & 16,6 & 13 h 30 & $3 \mathrm{j} 18 \mathrm{~h}$ \\
\hline $9 \mathrm{~B}$ & $25 / 04 / 96$ & $40 \mathrm{~V}$ & 100 & 41 & 57,3 & 18,3 & - & 10,5 & $2 h 45$ & $2 \mathrm{~h} 45$ \\
\hline $10 \mathrm{~B}$ & $29 / 04 / 96$ & E & 97 & 27 & 66,3 & 18,54 & 0,78 & 18,4 & $3 \mathrm{~h} 30$ & $17 \mathrm{~h}$ \\
\hline $11 \mathrm{~B}$ & $30 / 04 / 96$ & $E$ & 162 & 68 & 53,1 & 18,51 & 0,78 & 10,2 & $13 \mathrm{~h}$ & $13 \mathrm{~h}$ \\
\hline $12 B$ & $2 / 05 / 96$ & E & 100 & 25 & 65,3 & 18,12 & 0,85 & 7,1 & $13 \mathrm{~h} 30$ & 2j $13 h 30$ \\
\hline $13 B$ & $8 / 05 / 96$ & $40 \mathrm{~V}$ & 60 & 12 & 68,3 & 18,51 & 0,83 & 4,2 & $14 \mathrm{~h} 30$ & $14 \mathrm{~h} 30$ \\
\hline $14 \mathrm{~B}$ & $9 / 05 / 96$ & $40 \mathrm{~V}$ & 102 & 42 & 45,1 & 18,17 & 0,82 & 11,8 & $14 \mathrm{~h}$ & $14 \mathrm{~h}$ \\
\hline $15 B$ & $15 / 05 / 96$ & $E$ & 50 & 11 & 78,0 & 18,68 & - & 8,4 & $16 h 10$ & $16 h 10$ \\
\hline
\end{tabular}




\section{Influence de la taille des poissons}

Les tailles des poissons lâchés dans le BMC et recapturés dans l'exutoire ont ét: analysées sur les 10 lots dont le pourcentage de recapture était inférieur ou égal à $80 \%$, ce qui correspond à 967 poissons lâchés et 674 recapturés. La répartition en classes d; taille (Figure 8) fait apparaître que la proportion de poissons de taille inférieure à $175 \mathrm{mr}:$ est significativement plus faible dans les lots recapturés que dans les lots lâché; (WILCOXON, $Z=2,19 ; p<0,05$ ).

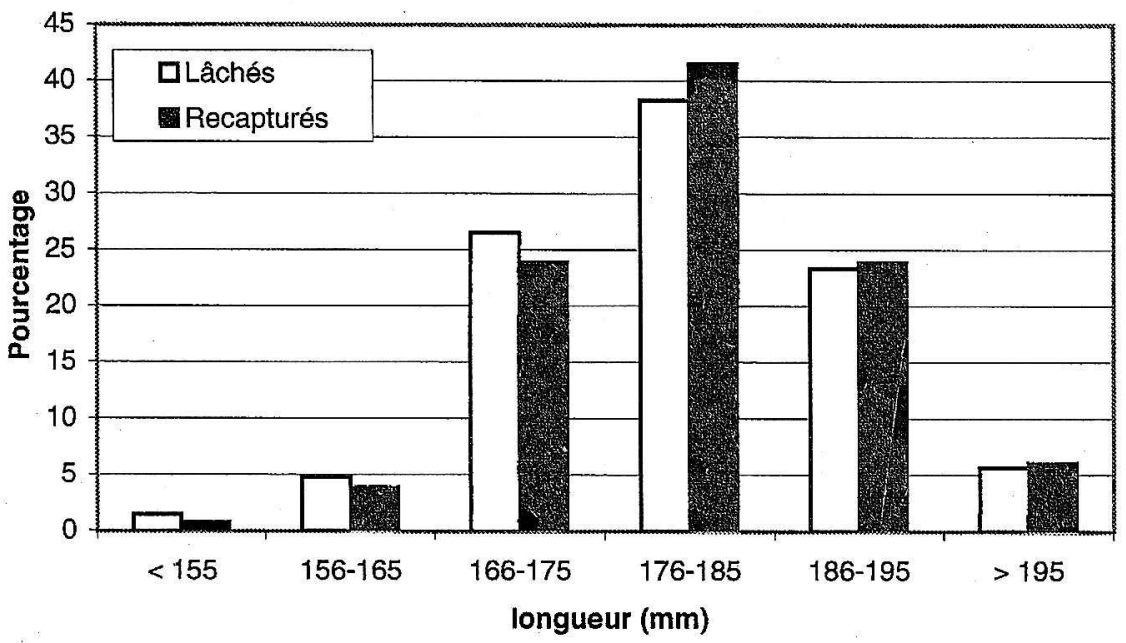

\section{Figure 8}

Répartition des tailles des smolts lâchés (lots BMC) et recapturés dans le piège.

\section{Figure 8}

Distribution of length of smolts released in the forebay ( BMC batches ») and trapped smolts.

\section{Comportement des smolts}

Les déplacements de 16 smolts ont pu être suivis par radio-pistage. Quinze d'entre eux, lâchés en amont de la retenue, sont arrivés par la galerie d'amenée et un a été lâché directement dans le BMC. Les principaux éléments recueillis sur leur comportement sont les suivants :

- dans la galerie d'amenée, les smolts semblent suivre plus ou moins passivement l'écoulement comme l'indique leur vitesse moyenne de progression $(1,6 \mathrm{~m} / \mathrm{s})$ très voisine de celle de l'écoulement de surface mesurée par lâchers de flotteurs. On note toutefois (Figure 9) une dispersion plus importante des vitesses de progression des smolts $(1,3$ à $1,8 \mathrm{~m} / \mathrm{s}$ ) que de celles des flotteurs ;

- arrivés dans le BMC, les smolts stoppent leur progression devant la grille de prise d'eau à l'exception d'un seul individu qui passe directement au travers de celle-ci ;

- les 15 smolts dont la progression a été stoppée par la grille, passent tous dans l'exutoire, soit une efficacité de l'exutoire pour les poissons radio-pistés de $94 \%$; 


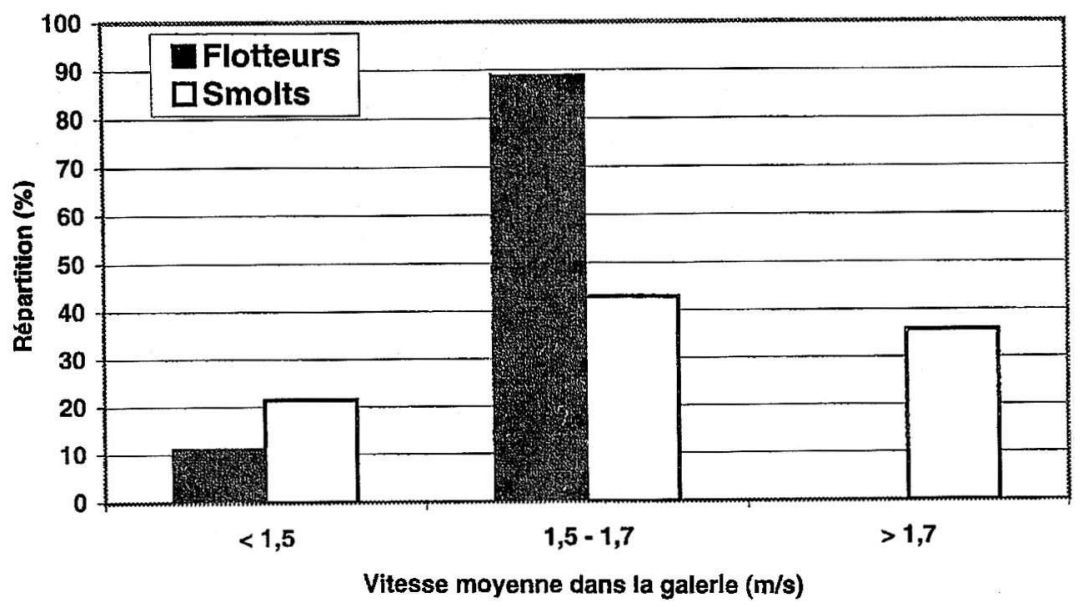

Figure 9

Distribution des vitesses moyennes des flotteurs et des smolts lors du trajet dans la galerie d'amenée de St. Cricq (2 500 mètres).

\section{Figure 9}

Distribution of mean velocities for floats and smolts during the passage through the head race $(2,500 \mathrm{~m})$.

- le point d'arrivée contre la grille du bassin se situe en rive gauche pour la majorité des smolts $(70 \%)$;

- la durée de stabulation dans le BMC est variable selon les individus (2 secondes à plus de 2 jours), mais est en général de courte durée puisque 7 smolts (44\%) restent moins de 20 secondes, $3(19 \%)$ stabulent entre 20 secondes et 5 minutes, $2(12 \%)$ restent entre 5 minutes et 1 heure, et 4 (25\%) stabulent dans le BMC plus d'une heure ;

- pendant leur présence dans le bassin, les smolts se déplacent en suivant les écoulements des zones de recirculation situées sur les deux rives et en effectuant des changements de rive devant la grille de prise d'eau ou bien stationnent dans les zones de contre-courant de chacune des rives. Quatre smolts sont remontés 1 à 12 fois dans la galerie après un premier séjour dans le bassin (durées totales comprises entre 20 minutes et $1 \mathrm{~h} 30$ ), mais cette progression reste limitée à quelques dizaines de mètres en amont du $\mathrm{BMC}$;

- la moitié des smolts rentre dans l'exutoire lors de leur premier passage devant l'entrée, et les autres après 2 ou 3 passages. Un seul smolt effectue 10 passages avant de pénétrer dans l'exutoire. On n'observe pas, par ailleurs, de stabulation de longue durée devant l'entrée de l'exutoire où, à l'exception d'un individu qui stationne durant une quarantaine de secondes, l'ensemble des smolts restent moins de 5 secondes ;

- les passages dans l'exutoire s'effectuent pour des débits compris entre $0,4 \mathrm{~m}^{3} / \mathrm{s}$ et $0,8 \mathrm{~m}^{3} / \mathrm{s}$, ce qui est représentatif de la gamme de variation des débits observés lors de l'expérimentation ;

- aucun smolt passé dans l'exutoire n'est ressorti du bassin tampon, par contre, les smolts mettent plusieurs minutes à franchir le seuil supportant la grille de piégeage (4 à 23 minutes pour 4 individus suivis manuellement). On a pu les observer s'approchant fréquemment de la crête du seuil, puis remonter de quelques mètres, le franchissement ne s'opérant qu'après plusieurs prospections ; 
- six smolts ont été présents au BMC lors du fonctionnement de l'éclairage situé à l'entrée de l'exutoire. Seuls deux individus ont subi plusieurs cycles d'éclairage, les quatre autres étant passés très rapidement dans l'exutoire $(t<20$ secondes) après leur arrivée dans le BMC. Ils ont tous les deux franchi l'exutoire lorsqu'il était éclairé.

\section{Efficacité du clapet associé à la barrière acoustique et comportement des smolts au barrage}

\section{Efficacité du clapet associé à la barrière acoustique}

\section{0 smolts répartis en 14 lots ont été déversés en amont du barrage.}

Six lots ont été déversés alors que la barrière acoustique était éteinte ( 0 volts), quatre lots alors qu'elle était en fonctionnement à faible puissance ( 20 volts) et quatre autres avec la barrière acoustique à forte puissance (40 volts).

Les résultats bruts de passage des smolts par le clapet sont rapportés dans le Tableau II. Les pourcentages de passage des smolts par le clapet (représentant l'efficacité du clapet et de la barrière acoustique associée) ont varié de $22,3 \%$ à $78 \%$ selon les lots, pour une moyenne de $52,4 \%$.

L'efficacité moyenne dans les 3 modes de fonctionnement de la barrière acoustique ( 0 volts, 20 volts et 40 volts) est rapportée dans le Tableau III. On a considéré d'une part, l'ensemble des lots (débit moyen du clapet compris entre 2 et $15 \mathrm{~m}^{3} / \mathrm{s}$ ), et d'autre part uniquement les lots soumis à un débit déversé au clapet compris entre 4 et $15 \mathrm{~m}^{3 / \mathrm{s}}$ de façon à éliminer les lots $(n=4)$ où il n'y a pas eu de déversement au clapet durant certaines périodes (passage obligatoire dans la galerie). On constate que l'efficacité moyenne dans ce dernier cas est de l'ordre de $60 \%$. On ne constate par contre aucun effet de la barrière acoustique sur les passages des smolts par le clapet qui ne diffèrent pas de façon significative selon les trois modes de fonctionnement tant sur l'ensemble des lots (KRUSKAL-WALLIS, $\mathrm{H}=0,08 ; \mathrm{p}=0,96$ ) que sur la série réduite (KRUSKAL-WALLIS, $H=2,0 ; p=0,37)$.

\section{Tableau III}

Pourcentage de smolts passant par le clapet selon le fonctionnement de la barrière acoustique.

\section{Table III}

Percentage of smolts passing over the flap gate at the dam in relation to operation of the acoustic barrier.

\begin{tabular}{ccc}
\hline $\begin{array}{c}\text { Fonctionnement de la barrière } \\
\text { acoustique }\end{array}$ & $\begin{array}{c}\text { Passages moyens par le } \\
\text { clapet }\left(2<\mathrm{Qc}<15 \mathrm{~m}^{3} / \mathrm{s}\right) \\
(14 \text { lots })\end{array}$ & $\begin{array}{c}\text { Passages moyens par le } \\
\text { clapet }\left(4<\mathrm{Qc}<15 \mathrm{~m}^{3} / \mathrm{s}\right) \\
(10 \text { lots }) \\
\%\end{array}$ \\
\hline Eteinte & $50,1 \pm 26,8$ & $65,7 \pm 16,2$ \\
Faible puissance (20 V) & $52,3 \pm 32,3$ & $62,2 \pm 12,8$ \\
Forte puissance (40 V) & $55,9 \pm 15,5$ & $56,9 \pm 28,9$ \\
\hline Moyenne & $52,4 \pm 11,2$ & $60,7 \pm 6,8$ \\
\hline
\end{tabular}


Le débit déversé au clapet durant l'expérimentation, du fait de sa variabilité 0 à $40 \mathrm{~m}^{3} / \mathrm{s}$ ) est très certainement un facteur prépondérant dans l'efficacité du dispositif de dévalaison. Nous avons reporté sur la Figure 10 le pourcentage de passage de smolts par le clapet selon le débit déversé au clapet et le mode de fonctionnement de la barrière dirant la période comprise entre le lâcher et la recapture de $90 \%$ des smolts piégés. On constate que les forts débits $\left(Q>8 \mathrm{~m}^{3} / \mathrm{s}\right)$ se traduisent par des efficacités importantes du ciapet $(40 \%$ à $80 \%$ ) sans que l'on ne puisse distinguer d'effet de la barrière acoustique. Pour les débits faibles $\left(\mathrm{Q}<5 \mathrm{~m}^{3} / \mathrm{s}\right)$, l'efficacité apparaît très variable ( $15 \%$ à $\left.70 \%\right)$ mais celte variabilité ne semble pas liée au fonctionnement de la barrière acoustique.

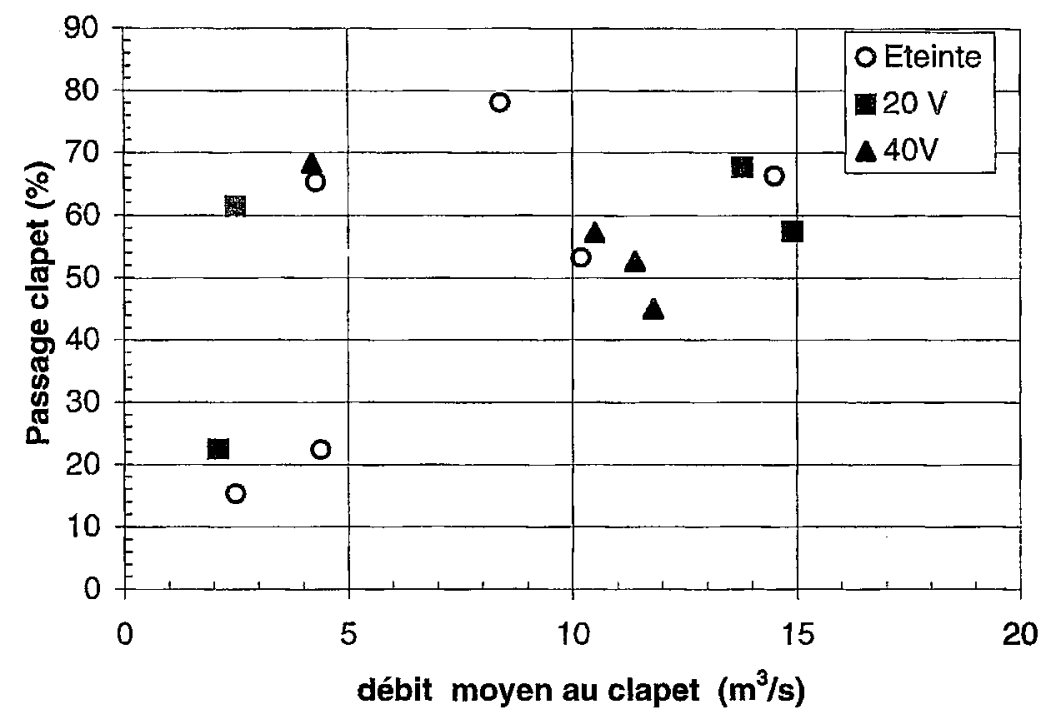

Figure 10

Pourcentage de smolts passant par le clapet du barrage en fonction du débit déversé et du fonctionnement de la barrière acoustique.

\section{Figure 10}

Percentage of smolts passing over the flap gate at the dam in relation to flap gate discharge and operation of the acoustic barrier.

\section{Comportement des smolts au voisinage du barrage et de la barrière acoustique}

28 smolts ont été lâchés en amont de la retenue de St. Cricq, dont 11 alors que la barrière acoustique était à l'arrêt ( 0 volts), 6 alors qu'elle était en fonctionnement à faible puissance ( 20 volts) et 11 pour un fonctionnement à forte puissance (40 volts). Les débits du gave lors de l'arrivée des smolts dans la retenue sont compris entre 12 et $42 \mathrm{~m}^{3} / \mathrm{s}$ et les débits déversés au clapet entre 0 et $22 \mathrm{~m}^{3} / \mathrm{s}$. Le débit turbiné était maximal $\left(19 \mathrm{~m}^{3} / \mathrm{s}\right)$ lors de l'arrivée de tous les smolts, à l'exception d'un seul individu pour lequel le débit était de $11 \mathrm{~m}^{3} / \mathrm{s}$.

Les principaux éléments relatifs au comportement des smolts sont les suivants :

- la durée de présence des poissons dans la retenue avant leur passage par le clapet ou par la galerie varie de 1 minute à 18 heures, mais est en général de courte durée, puisque la moitié des smolts (14 individus) y reste moins de 2 minutes, 11 y restent entre 2 et 20 minutes, et 3 smolts seulement y passent plus de 20 minutes; 
- en l'absence de fonctionnement de la barrière acoustique, les trajets des smols dans la retenue se sont avérés dépendants du débit déversé par le clapet : passage dire:t par le clapet en longeant la rive gauche de la retenue lorsque le débit déversé est élevá ( 1 seul poisson observé pour un débit de $22 \mathrm{~m}^{3} / \mathrm{s}$ ), passage direct par la galerie $\mathrm{\epsilon}$ longeant la rive droite ou en passant par le milieu de la retenue lorsque le débit déveré est faible $\left(<3 \mathrm{~m}^{3} / \mathrm{s}\right)$ et trajets très variables suivant les individus pour les débi:s intermédiaires $\left(6 \mathrm{~m}^{3} / \mathrm{s}<\mathrm{Q}<15 \mathrm{~m}^{3} / \mathrm{s}\right)$;

- lorsque la barrière acoustique fonctionne à faible puissance (20 volts), on ne remarque pas de différence notable dans les trajets des 6 smolts dans la retenue par rapport a celui observé sans la barrière acoustique. Par contre, avec la barrière à forte puissance (40 volts), on remarque pour 3 poissons (sur 11 individus soumis à cette configuration) ure déviation vers la rive gauche qui ne semble pas imputable aux conditions hydrodynamiques ;

- les durées de stabulation dans les zones les plus proches des émetteurs sonores (zones " prise d'eau », Figure 4), sont de courte durée qu'elle que soit l'ambiance sonore (2 à 13 secondes avec la barrière arrêtée, 8 à 80 secondes avec la barrière 20 volts et 3 à 37 secondes avec la barrière 40 volts) et ne diffèrent pas statistiquement selon les trois conditions de fonctionnement de la barrière (KRUSKALL-WALLIS, $H=3,64 ; p=0,16$ ). Le comportement des smolts dans les zones à forte puissance sonore (120 à $130 \mathrm{~dB}$ ), où l'on aurait pu penser constater les réactions les plus marquées, ne semble donc pas différer de celui observé lorsque la barrière est arrêtée ;

- globalement, c'est dans la zone clapet que les smolts stationnent le plus longuement (Figure 4B). Ces stabulations, avant passage par le clapet, sont de durées très variables (10 secondes à $1 \mathrm{~h} 30$ ), et apparaissent d'autant plus élevées que le débit déversé est faible: plusieurs minutes (2 minutes à 1 h 30 ) lorsque les débits sont inférieurs à $10 \mathrm{~m} / \mathrm{s}$, et de 10 secondes à 1 minute pour les débits supérieurs. Des observations visuelles effectuées sur des bancs de smolts dévalant ont montré une réaction de fuite vers l'amont à l'approche de la crête du clapet. La plupart des smolts (13 sur 15) venus à proximité immédiate du clapet y passent (débits déversés compris entre $6,5 \mathrm{~m}^{3} / \mathrm{s}$ et $\left.23 \mathrm{~m}^{3} / \mathrm{s}\right)$.

La répartition globale des passages de smolts radio-pistés entre le clapet et la galerie d'amenée, tous fonctionnements de la barrière acoustique confondus, est respectivement de $13(46 \%)$ et $15(54 \%)$ individus.

Les passages par le clapet selon le mode de fonctionnement de la barrière acoustique et pour trois catégories de débit sont rapportés sur la Figure 11 et dans le Tableau IV. Selon la gamme de débit, les passages par le clapet sont compris entre $36 \%$ et $50 \%$ lorsque la barrière est éteinte, $50 \%$ lorsque la barrière fonctionne à faible puissance et $56 \%$ à $62 \%$ avec la barrière à forte puissance. Les pourcentages de passage semblent légèrement plus élevés avec la barrière à forte puissance, mais sont cependant à considérer avec prudence car ils s'appuient sur de faibles nombres de poissons (4 à 11 individus par situation). 
Tósleau IV

Zone de passage des smolts radio-pistés (galerie ou clapet) et pourcentage de passage par le clapet pour trois gammes de débit déversé et selon le fonctionnement de la barrière acoustique.

Thle IV

Location of smolt passage (flap gate or head race) and percentage of flap gate passages in relation to flap gate discharge and operation of the acoustic barrier.

\begin{tabular}{ccccc}
\hline $\begin{array}{c}\text { Débit déversé au } \\
\text { clapet (m3/s) }\end{array}$ & \multicolumn{3}{c}{ Barrière acoustique } \\
\hline & & Eteinte & Faible puissance (20 V) & Forte puissance (40 V) \\
\hline \multirow{2}{*}{$0<\mathrm{Qc}<23$} & Passage Galerie (Nb) & 7 & 3 & 5 \\
& Passage Clapet (Nb) & 4 & 3 & 6 \\
& Clapet \% & $36 \%$ & $50 \%$ & $55 \%$ \\
\hline \multirow{2}{*}{$5<\mathrm{Qc}<23$} & Passage Galerie (Nb) & 4 & 2 & 5 \\
& Passage Clapet (Nb) & 3 & 2 & 5 \\
& Clapet \% & $43 \%$ & $50 \%$ & 3 \\
\hline \multirow{2}{*}{$5<$ Qc $<12$} & Passage Galerie (Nb) & 3 & 2 & 5 \\
& Passage Clapet (Nb) & 3 & 2 & $62 \%$ \\
\hline
\end{tabular}

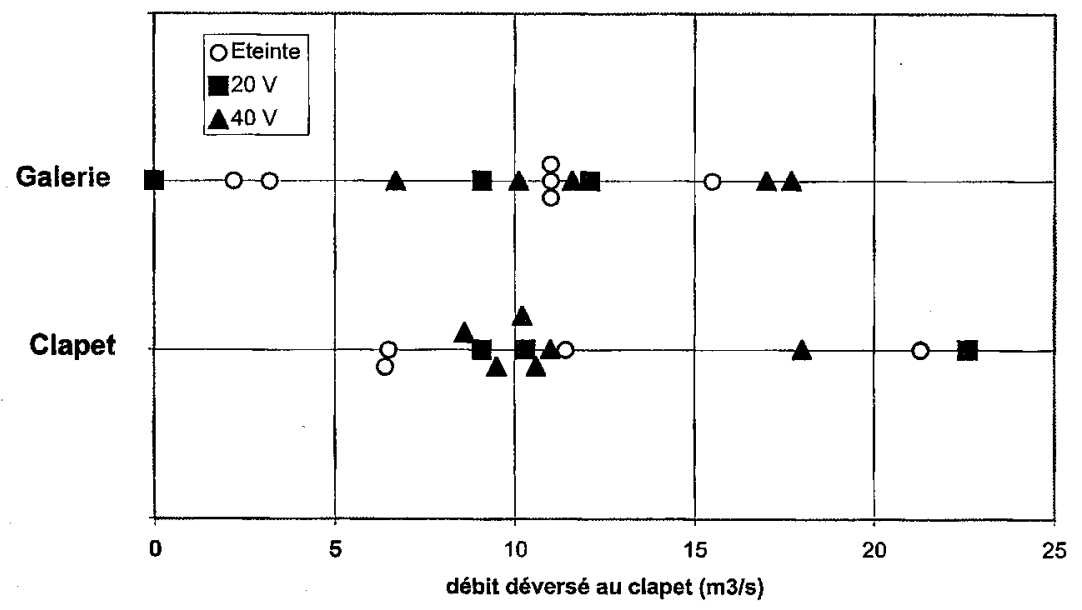

Figure 11

Zone de passage des smolts radio-pistés au barrage de St. Cricq en fonction du débit déversé au clapet et du fonctionnement de la barrière acoustique.

\section{Figure 11}

Location of passage of radiotagged smolts at St. Cricq dam in relation to flap gate discharge and operation of the acoustic barrier.

Pour tenter de quantifier l'effet de la barrière acoustique sur le comportement des smolts, on a analysé la durée totale de présence des poissons sur le site (pour détecter un éventuel ralentissement de la migration dû à l'effet répulsif de la barrière) et le pourcentage du temps passé sur la rive gauche de la retenue par rapport au temps de présence sur le site, dans la mesure où l'effet escompté de la barrière est de canaliser les smolts vers la rive gauche. Les résultats font apparaître une stabulation de plus longue durée sur le site lorsque la barrière à forte puissance fonctionne (Figure 12), mais cette augmentation n'est cependant pas statistiquement significative (KRUSKALL-WALLIS, $H=2,7 ; p=0,26$ ). Par contre, on n'observe pas de stabulation plus élevée en rive gauche lorsque la barrière fonctionne que lorsqu'elle est arrêtée (Figure 13) (KRUSKALL-WALLIS, $H=0,69 ; p=0,71$ ). 


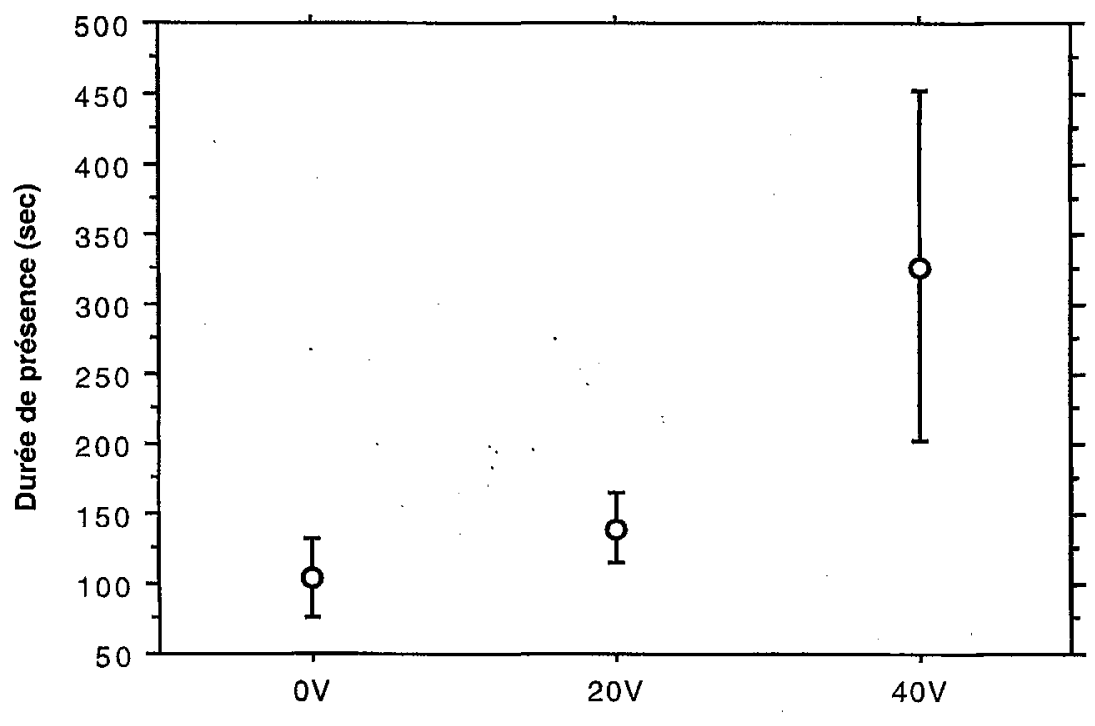

Fonctionnement de la barrière acoustique

Figure 12

Durée de présence des smolts radio-pistés dans la retenue de St. Cricq selon le fonctionnement de la barrière acoustique.

\section{Figure 12}

Residence time of radiotagged smolts in the St. Cricq impoundment in relation to operation of the acoustic barrier.

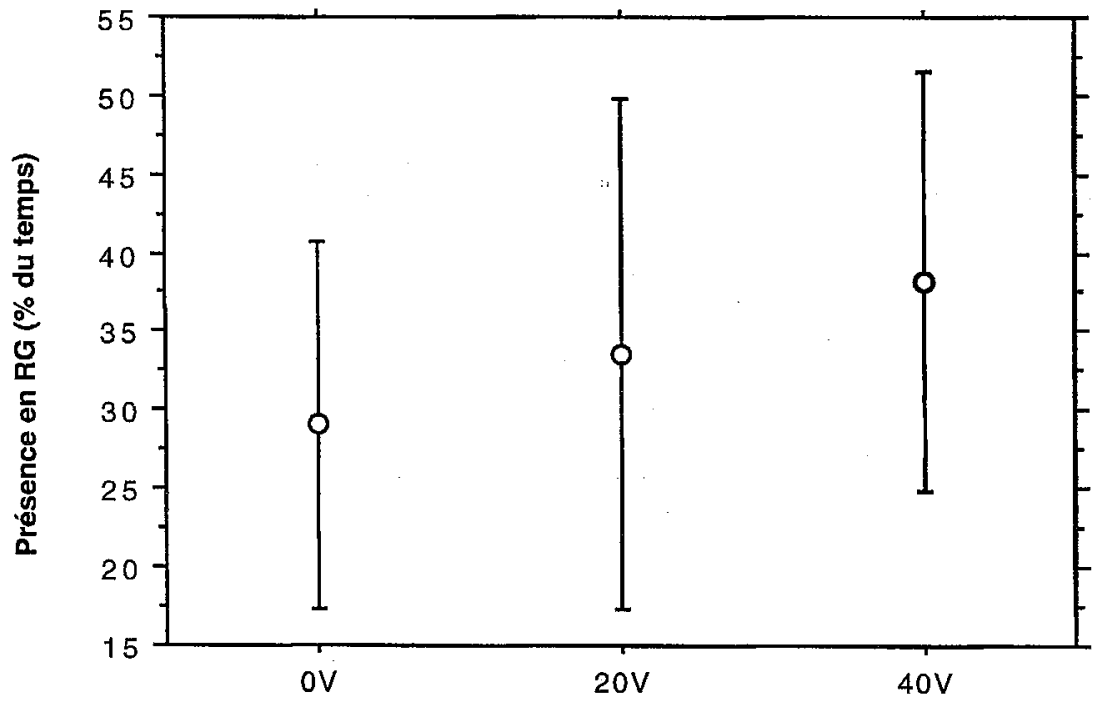

Fonctionnement de la barrière acoustique

Figure 13

Durée de stabulation des smolts radio-pistés en rive gauche de la retenue di St. Cricq (en pourcentage du temps total de présence dans la retenue).

Figure 13

Residence time of radiotagged smolts on the left bank of the St. Crict impoundment (as a percentage of total residence time in the impoundment). 


\section{DISCUSSION}

La première partie des résultats de cette expérimentation a trait à l'étude de l'eificacité, pour les smolts de saumon atlantique, d'un exutoire de dévalaison associé aux grilles de prises d'eau d'une centrale hydroélectrique. Le principe de fonctionnement d'un tel dispositif repose sur le pouvoir répulsif de la grille de prise d'eau qui doit stopper momentanément la progression des migrateurs, et sur les capacités d'attraction d'un exutoire situé à proximité (TRAVADE et LARINIER, 1992 ; LARINIER et TRAVADE, 1997).

Le pouvoir répulsif de la grille de prise d'eau (BMC) est bien mis en évidence par l'efficacité élevée de l'exutoire et par le comportement des poissons radio-pistés : ceux-ci devalent à une vitesse voisine de l'écoulement dans la galerie en amont du BMC et s'arrêtent tous devant la grille à l'exception d'un seul individu (sur 16 poissons) qui passe directement au travers.

L'efficacité de l'exutoire situé au BMC est d'environ $80 \%$. Ce dispositif est le plus performant des dispositifs de ce type testés ces dernières années en France sur les sites de Soeix (LARINIER et TRAVADE, 1996), Bedous (CHANSEAU, LARINIER et TRAVADE, 1999), Camon (CROZE, CHANSEAU et LARINIER, 1999) ou Halsou (GOSSET et al., 1998 ; GOSSET et TRAVADE, 1999) où les efficacités sont comprises entre $55 \%$ et $73 \%$. Le débit détourné dans l'exutoire $(1,5 \%$ à $4,2 \%$ du débit turbiné) est comparable à celui utilisé dans ces autres dispositifs et se situe dans la gamme de $2 \%$ à $10 \%$ du débit turbiné préconisée en France (LARINIER et TRAVADE, 1997). Cette bonne efficacité semble essentiellement attribuable à la répulsivité de la grille et aux conditions hydrodynamiques avoisinant la prise d'eau et l'entrée de l'exutoire. La répulsivité de la grille de prise d'eau est vraisemblablement plus élevée que celle des autres sites testés en raison du faible écartement des barreaux $(2,5 \mathrm{~cm}$ au lieu de 3 à $4 \mathrm{~cm}$ sur les autres usines) et de la composante de la vitesse d'écoulement tangentielle à la grille dont la valeur est très élevée $(0,65$ à $0,8 \mathrm{~m} / \mathrm{s})$. Grâce à cette vitesse élevée, les smolts sont guidés très rapidement (quelques secondes) vers la berge où est situé l'exutoire.

Les conditions hydrauliques à l'entrée de l'exutoire, dont l'importance a été démontrée lors des études précédemment évoquées, apparaissent également très favorables par le fait que l'accélération à l'entrée de l'exutoire est faible, la vitesse à l'entrée de l'exutoire restant du même ordre de grandeur que celle de l'écoulement à són approche, et que la profondeur d'eau y est importante $(0,8$ à $0,95 \mathrm{~m})$. Cela résulte de la présence d'un bassin tampon à l'aval de l'exutoire (chute comprise entre 3 et $10 \mathrm{~cm}$ ) et de la forme du seuil (seuil épais). L'importance de ces facteurs semble confirmée par les hésitations des smolts à passer sur le déversoir mince du piège et par le clapet du barrage où l'accélération est élevée et la profondeur d'eau peu importante.

Comme observé sur les autres sites, on constate une efficacité de l'exutoire moindre pour les smolts de taille inférieure à $17,5 \mathrm{~cm}$. L'explication peut résider dans la répulsivité de la grille de prise d'eau moindre pour les individus de petite taille et/ou par leurs capacités de nage limitées. Cette sélectivité peut expliquer les meilleurs résultats d'efficacité de l'exutoire obtenus avec les poissons radiomarqués (94\%) qu'avec le marquage-recapture $(79 \%)$, dans la mesure où les poissons munis d'émetteurs étaient tous d'une taille supérieure à $18 \mathrm{~cm}$.

Les effets de l'éclairage de l'exutoire sont difficiles à appréhender compte tenu de la rapidité avec laquelle les smolts rentrent dans l'exutoire. 
La barrière acoustique s'est avérée peu voire pas efficace pour détourner les smoin de la prise d'eau du barrage et les repousser vers le clapet de décharge. Le passa! ! d'environ $50 \%$ à $60 \%$ des poissons par ce clapet résulte, en effet, essentiellement l'importance du débit déversé ( $8 \mathrm{~m}^{3} / \mathrm{s}$, soit $45 \%$ du débit turbiné en valeur modale), ? aucune différence statistique des passages n'est observée entre les situations où i barrière acoustique ne fonctionne pas et celles où elle fonctionne à faible ou à fol: puissance. Dans les rares cas où ce débit est resté inférieur à $5 \mathrm{~m}^{3} / \mathrm{s}$ (3 lots), le passa it par le clapet a été très variable $(20 \%$ à $70 \%)$ mais sans influence notable de la barriè : acoustique. Le suivi des poissons par radio-pistage semble montrer quelques modificatio $1:$ de comportement sous l'effet de la barrière à forte puissance (éloignement momentané la prise d'eau), mais ces constatations sont limitées à quelques individus. En tout état it cause, le stimulus sonore répulsif, s'il existe, n'apparaît pas de nature à concurrencer façon substantielle le stimulus hydraulique. Ces résultats sont en accord avec ceux obten: à la centrale d'Halsou sur la Nive (GOSSET et TRAVADE, 1999) où une barrière acoustiq:! associée à un rideau de bulles (système BAFF de FGS) s'est avérée totalement inefficai, pour détourner les smolts vers un exutoire. On peut se poser la question de savoir si i: puissance sonore était suffisante ou si la gamme des fréquences sonores utilisée par $F G_{i}$ i $(100-600 \mathrm{~Hz})$ est effectivement répulsive pour les salmonidés. II semblerait, en effet, qui les fréquences les plus répulsives pour les smolts de saumon pourraient se situer dan une gamme beaucoup plus basse $(10 \mathrm{~Hz})$ comme l'ont montré KNUDSEN et al. (199? 1994) lors d'essais en laboratoire et sur le terrain.

\section{CONCLUSION}

Cette étude a permis de comparer deux dispositifs de dévalaison pour les smolts $d$ saumon atlantique. L'efficacité de l'exutoire de surface associée à la grille de prise d'eat de l'ordre de $80 \%$, peut être considérée comme bonne en regard des autres dispositifs d ce type testés par ailleurs. Les facteurs tels que l'espacements des barreaux $\epsilon$ l'hydrodynamique devant le plan de grille et à l'entrée de l'exutoire apparaisser prépondérants pour ce type de dispositif.

Par contre, la capacité de la barrière acoustique à détourner les smolts de la pris d'eau s'est avérée très faible voire nulle. Il ne nous a cependant pas été possible de savo quelles étaient les causes de cette inefficacité : puissance insuffisante du champ sonore? gamme de fréquence insuffisamment répulsive pour le poisson?, imprécision du guidag des poissons par le champ sonore?

Le suivi du comportement des poissons au voisinage de ces deux dispositifs nou confirme l'extrême importance du facteur hydrodynamique comme stimulus primordic d'orientation du poisson en dévalaison. II nous apparaît donc essentiel d'associer à tout «barrière comportementale » quelle qu'elle soit, les conditions hydrodynamiques les plu favorables au guidage des poissons vers un exutoire.

\section{REMERCIEMENTS}

Cette étude a bénéficié de l'aide technique de J.M. Bach, C. Brugel, L. Drouadaint E. Galiay, R. Galland et J. Néau.

Nous remercions le CSP pour la mise à disposition de smolts, la FDPPMA de Pyrénées Atlantiques et le personnel des piscicultures de Pedehourat et de Préchac qui $e$ ont assuré la stabulation et le personnel du groupement d'Asasp d'EDF pour son aid précieuse lors de la réalisation des opérations de terrain. 


\section{BISLIOGRAPHIE}

BAGLINIERE J.L., 1976. Etude des populations de Saumon atlantique (Salmo salar L. 1766) en Bretagne-Basse-Normandie. 1 - Caractéristiques des smolts de la rivière Ellé. Ann. Hydrobiol., 7, 141-158.

BOUSQUET B., 1979. Biologie et migration des smolts de saumon atlantique (Salmo salar L.) dans les bassins Loire-Allier et Adour-Gave d'Oloron. Thèse de Doctorat Inst. Nat. Polyrtech., Toulouse, France, $67 \mathrm{p}$.

C.ANSEAU M., LARINIER M., TRAVADE F., 1999. Efficacité d'un exutoire de dévalaison pour smolts de saumon atlantique (Salmo salar L.) et comportement des poissons au niveau de l'aménagement hydroélectrique de Bedous sur le gave d'Aspe étudiés par le technique de marquage-recapture et par radiotélémétrie. Bull. Fr. Pêche Piscic.,353/354, 99-120.

CAOZE O., CHANSEAU M., LARINIER M., 1999. Efficacité d'un exutoire de dévalaison pour smolts de saumon atlantique (Salmo salar L.) et comportement des poissons au niveau de l'aménagement hydroélectrique de Camon sur la Garonne. Bull. Fr. Pêche Piscic., 353/354, 121-140.

EPRI, 1988. Proceedings : Fish protection at steam and hydroelectric power plants. EPRI CS/EA/AP-5663-SR, $350 \mathrm{p}$.

EPRI, 1994. Research update of fish protection technologies for water intakes. EPRI TR-104122, $208 \mathrm{p}$.

FERGUSON J.W., POE T.P., CARLSON T.J., 1998. Surface-oriented bypass systems for juvenile salmonids on the Colombia River, USA. In: JUNGWIRTH M., SCHMUTZ S. and WEISS S. (Eds.), Fish migration and fish bypasses, Fishing News Books, 281-299.

GOSSET C., TRAVADE F., LABASTIE C., GARAICOECHEA C., 1998. Etude de dispositifs de dévalaison pour smolts de saumon atlantique : influence de la lumière sur l'efficacité des exutoires de dévalaison. Centrale hydroélectrique de Halsou (Nive -64). INRA, Station d'hydrobiologie de Saint-Pée-sur-Nivelle, 34 p.

GOSSET C., TRAVADE F., 1999. Etude de dispositifs d'aide à la migration de dévalaison : barrières comportementales. Journée annuelle de la Société Française d'Ichtyologie. Cybium, special issue, (in press).

INGENDAHL D., BACH J.M., LARINIER M., TRAVADE F., 1996. The use of telemetry in studying downstream migration of Atlantic salmon smolts at a hydro-electric power plant in South-West France: preleminary results. In : Underwater Biotelemetry, Proc. of the First Conf. and Workshop on Fish Telemetry in Europe, BARAS E. and PHILIPPART J.C. (Eds.), Univ. of Liège, 121-128.

KNUDSEN F.R., ENGER P.S., SAND O., 1992. Awareness reactions and avoidance responses to sound in juvenile Atlantic salmon, Salmo salar L. Jour. of Fish Biol., 40, 523-534.

KNUDSEN F.R., ENGER P.S., SAND O., 1994. Avoidance responsies to low frequency sounds in downstream migrating Atlantic salmon smolt, Salmo salar. Jour. of Fish Biol., 45, 227-233.

LARINIER M., BOYER-BERNARD S., 1991a. Dévalaison des smolts et efficacité d'un exutoire de dévalaison à l'usine hydroélectrique d'Halsou sur la Nive. Bull. Fr. Pêche Piscic., 321, 72-92.

LARINIER M., BOYER-BERNARD S., 1991b. La dévalaison des smolts de saumon atlantique au barrage de Poutès sur l'Allier (43) : utilisation de lampes à vapeur de mercure en vue d'optimiser l'efficacité d'un exutoire de dévalaison. Bull. Fr. Pêche Piscic., 323, 129-148. 
LARINIER M., TRAVADE F., 1996. Smolt behavior and downstream fish bypass efficiency at small hydroelectric plants in France. Association Internationale de Recherches Hydrauliques, 2 ème Symposium international sur l'hydraulique et les habitats, Ecohydraulique 2000, Québec, vol B, 891-902.

LARINIER M., TRAVADE F., 1997. The development and evaluation of downstrean bypasses for juvenile salmonids at small hydroelectric plants in France. Americen Fisheries Society, 127th Annual Meeting - Monterey, California, August 24-28 199:, $18 \mathrm{p}$.

LOEFFELMAN P.H., VAN HASSEL J.H., KLINECT D.A., 1991. Using sound to divert fict? from turbine intakes. Hydro Review, Octobre 1991, 30-43.

McCORMICK S.D., HANSEN L.P., QUINN T.P., SAUNDERS R.L., 1998. Movement, migration, and smolting of Atlantic salmon (Salmo salar). Can. J. Fish. Aquat. Sc:, 55(suppl. 1), 77-92.

SUBACOUSTECH, 1996a. Acoustical modelling of the proposed fish deterrency systein for installation at St. Cricq. Rapport CNA232R0101, $31 \mathrm{p}$.

SUBACOUSTECH, 1996b. Acoustic survey of St. Cricq hydroelectric power station. Rapport CNA232R0202, $11 \mathrm{p}$.

TAFT N., 1988. Evaluations of fish protection systems for use at hydroelectric plants. Hydro Review, 54-62.

THORPE J.F., 1981. Migration in salmonids with special reference to juvenile movements in freshwater. In: BRANNON E.L. and SALO E.O. (Eds.), Salmon and trout migratory behavior symposium, 86-97.

TRAVADE F., LARINIER M., 1992. La migration de dévalaison: problèmes et dispositifs. Bull. Fr. Pêche Piscic., 326-327, 165-176.

TURNPENNY A.W.H., THATCHER R.W., LOEFFELMAN P.H., 1993. Experiments on the use of sound as a fish deterrent. Rapport Fawley Aquatic Research Laboratories FCR040/93.

TURNPENNY A.W.H., 1995. Diversion of downstream migrating fish at hydroectric facilities: scope for using acoustic deflecting method at EDF's St. Cricq, Bedous and Halsou plants. Rapp. Expertise FGS. 10 p. 